\title{
NONLINEAR NONCOERCIVE NEUMANN PROBLEMS WITH A REACTION CONCAVE NEAR THE ORIGIN
}

\author{
Pasquale Candito - Giuseppina D'Aguí \\ Nikolaos S. Papageorgiou
}

\begin{abstract}
We consider a nonlinear Neumann problem driven by the $p$ Laplacian with a concave parametric reaction term and an asymptotically linear perturbation. We prove a multiplicity theorem producing five nontrivial solutions all with sign information when the parameter is small. For the semilinear case $(p=2)$ we produce six solutions, but we are unable to determine the sign of the sixth solution. Our approach uses critical point theory, truncation and comparison techniques, and Morse theory.
\end{abstract}

\section{Introduction}

Let $\Omega \subseteq \mathbb{R}^{N}$ be a bounded domain with a $C^{2}$-boundary $\partial \Omega$. In this paper, we study the following nonlinear Neumann problem:

$$
\begin{cases}-\Delta_{p} u+\beta(z)|u|^{p-2} u=\lambda|u|^{q-2} u+f(z, u) & \text { in } \Omega, \\ \frac{\partial u}{\partial n}=0 & \text { on } \partial \Omega,\end{cases}
$$

for $\beta \in L^{\infty}(\Omega), \beta(z) \geq 0$ almost everywhere in $\Omega$, with $\beta \neq 0, \Delta_{p} u:=$ $\operatorname{div}\left(\|\nabla u\|^{p-2} \nabla u\right)$ the $p$-Laplacian operator, $1<p<+\infty$, and $n(\cdot)$ the outward unit normal on $\partial \Omega$. Moreover, $\lambda>0$ is a parameter and $q \in(1, p)$. So, the term $\lambda|x|^{q-2} x$ is strictly sublinear (concave term). In addition, we assume that the perturbation $f(z, x)$ is a Carathéodory function (i.e. for all $x \in \mathbb{R}$,

2010 Mathematics Subject Classification. Primary: 35J25; Secondary: 35J80, 58E05.

Key words and phrases. Concave term; constant sign solutions; nodal solutions; nonlinear regularity; critical groups. 
$z \mapsto f(z, x)$ is measurable and for almost all $z \in \Omega, x \mapsto f(z, x)$ is continuous) which exhibits $(p-1)$-linear growth near $\pm \infty$.

Our aim in this paper is to prove a multiplicity theorem for problem $\left(\mathrm{P}_{\lambda}\right)$ for certain values of the parameter $\lambda$ and provide sign information for all solutions produced. More precisely, we show that for all parameters $\lambda>0$ suitably small, problem $\left(\mathrm{P}_{\lambda}\right)$ has at least five nontrivial smooth solutions, four of constant sign (two positive and two negative, Proposition 3.6) and the fifth is nodal (Theorem 4.3). In the semilinear case $(p=2)$, we produce six nontrivial smooth solutions, but we are unable to determine the sign of the sixth solution (Theorem 5.2).

In the past, problems with concave terms and asymptotically linear perturbations were studied primarily in the context of semilinear $(p=2)$ Dirichlet equations. We mention the works of de Paiva and Massa [4], Hu and Papageorgiou [12], Li, Wu and Zhou [16], Perera [25] and Wu and Yang [27]. Extensions to the Dirichlet $p$-Laplacian can be found in Guo and Zhang [10] (for $p \geq 2$ ), Gasinski and Papageorgiou [8] (singular problems), [9] (positive solutions of anisotropic problems), Kyritsi and Papageorgiou [14] (pairs of positive solutions) and Motreanu, Motreanu and Papageorgiou [19] (problems which have an asymmetric reaction, superlinear in the positive direction and coercive in the negative direction; this leads to a different geometry and a distinct multiplicity theorem with respect to our framework). For Neumann equations we mention the work of Motreanu, Motreanu and Papageorgiou [20], who study the equation

$$
-\Delta_{p} u+\lambda|u|^{p-2} u=f(z, u) \quad \text { in } \Omega, \quad \frac{\partial u}{\partial n}=0 \quad \text { on } \partial \Omega .
$$

Here $\lambda>0$ is a parameter and $f(x, z)$ is a Carathéodory reaction exhibiting a concave term near zero. A multiplicity result is proved (including a nodal solution) for $\lambda>0$ small (Theorem 4.3).

We should also mention the work of Motreanu, Motreanu and Papageorgiou [21] which inspired our results on the semilinear case (see Section 5).

Our approach uses critical point theory, combined with suitable truncation and comparison techniques, and with Morse theory (critical groups).

The paper is arranged as follows. In the next section, for the convenience of the reader, we recall the main mathematical tools that we use in this work. Section 3 is devoted to constant sign solutions for $\left(\mathrm{P}_{\lambda}\right)$, Section 4 to the existence of a nodal solution, and the semilinear case is studied on Section 5 .

\section{Mathematical background}

Let $X$ be a Banach space and $X^{*}$ its topological dual. By $\langle\cdot, \cdot\rangle$ we denote the duality brackets for the pair $\left(X^{*}, X\right)$. Given $\phi \in C^{1}(X)$, we say that $\phi$ satisfies the "Cerami condition" (the "C-condition" for short), if the following is 
true: "Every sequence $\left\{x_{n}\right\}_{n \geq 1} \subseteq X$ such that $\left\{\phi\left(x_{n}\right)\right\}_{n \geq 1} \subseteq \mathbb{R}$ is bounded and

$$
\left(1+\left\|x_{n}\right\|\right) \phi^{\prime}\left(x_{n}\right) \rightarrow 0 \quad \text { in } X^{*} \text { as } n \rightarrow \infty,
$$

admits a strongly convergent subsequence".

This compactness-type condition is in general weaker than the usual PalaisSmale condition. Nevertheless, it is strong enough to prove a deformation theorem and from it to derive the minimax theory of certain critical values of $\phi$. In particular, we have the following result, known in the literature as the "mountain pass theorem".

TheOREM 2.1. If $X$ is a Banach space, $\varphi \in C^{1}(X), x_{0}, x_{1} \in X, \rho>0$, $\left\|x_{0}-x_{1}\right\|>\rho$,

$$
\begin{gathered}
\max \left\{\varphi\left(x_{0}\right), \varphi\left(x_{1}\right)\right\}<\inf \left\{\varphi(x):\left\|x-x_{0}\right\|=\rho\right\}=\eta_{\rho}, \\
c=\inf _{\gamma \in \Gamma} \max _{0 \leq t \leq 1} \varphi(\gamma(t))
\end{gathered}
$$

where $\Gamma=\left\{\gamma \in C([0,1], X): \gamma(0)=x_{0}, \gamma(1)=x_{1}\right\}$ and $\varphi$ satisfies the $C$ condition, then $c \geq \eta_{\rho}$ and $c$ is a critical value of $\varphi$.

Important in our analysis of problem $\left(\mathrm{P}_{\lambda}\right)$ will be the Banach space $C^{1}(\bar{\Omega})$. This is an ordered Banach space with positive cone

$$
C_{+}=\left\{u \in C^{1}(\bar{\Omega}): u(z) \geq 0, \text { for all } z \in \bar{\Omega}\right\} .
$$

This cone has nonempty interior given by

$$
\operatorname{int} C_{+}=\left\{u \in C_{+}: u(z)>0 \text {, for all } z \in \bar{\Omega}\right\} .
$$

We denote by $\|\cdot\|_{p}$ the usual norm on $L^{p}(\Omega)$ and by $\|\cdot\|$ that on $W^{1, p}(\Omega)$ defined by

$$
\|u\|=\left(\|D u\|_{p}^{p}+\|u\|_{p}^{p}\right)^{1 / p}, \quad \text { for all } u \in W^{1, p}(\Omega) .
$$

The same notation will also be used to denote the norm in $\mathbb{R}^{N}$. However, no confusion is possible, since it will always be clear from the context which norm we use.

Now, we introduce some basic definitions and results concerning the following Neumann problem:

$$
\begin{cases}-\Delta_{p} u+\beta(z)|u|^{p-2} u=l(z, u) & \text { in } \Omega, \\ \frac{\partial u}{\partial n}=0 & \text { on } \partial \Omega,\end{cases}
$$

where $1<p<+\infty$ and $l: \Omega \times \mathbb{R} \rightarrow \mathbb{R}$ is a Carathéodory function with subcritical growth with respect to the second variable, i.e.

$\left(1^{*}\right)$ there exist a nonnegative and nontrivial function $\alpha \in L^{\infty}(\Omega)$ and $1<$ $r<p^{*}$, such that

$$
|l(z, x)| \leq \alpha(z)\left(1+|x|^{r-1}\right), \quad \text { for a.a. } z \in \Omega \text { and all } x \in \mathbb{R},
$$


where $p^{*}=p N /(N-p)$, if $p<N$ and $p^{*}=+\infty$, if $p \geq N$.

A weak solution of problem $\left(\mathrm{P}_{1}\right)$ is any function $u \in W^{1, p}(\Omega)$ such that

$$
\int_{\Omega}\left[\|D u\|^{p-2}(D u, D v)_{\mathbb{R}^{N}}+\beta(z)|u|^{p-2} u v-l(z, u) v\right] d z=0,
$$

for $v \in W^{1, p}(\Omega)$. We recall that $\underline{u} \in W^{1, p}(\Omega)$ is a sub-solution, while $\bar{u} \in$ $W^{1, p}(\Omega)$ is a super-solution for problem $\left(\mathrm{P}_{1}\right)$, if $\partial \underline{u} / \partial n \leq 0 \leq \partial \bar{u} / \partial n$ on $\partial \Omega$, and

$$
\begin{aligned}
& \int_{\Omega}\left[\|D \underline{u}\|^{p-2}(D \underline{u}, D v)_{\mathbb{R}^{N}}+\beta(z)|\underline{u}|^{p-2} \underline{u} v-l(z, \underline{u}) v\right] d z \leq 0, \\
& \int_{\Omega}\left[\|D \bar{u}\|^{p-2}(D \bar{u}, D v)_{\mathbb{R}^{N}}+\beta(z)|\bar{u}|^{p-2} \bar{u} v-l(z, \bar{u}) v\right] d z \geq 0,
\end{aligned}
$$

for all $v \in W^{1, p}(\Omega), v \geq 0$ almost everywhere in $\Omega$, respectively. Set

$$
L(z, x)=\int_{0}^{x} l(z, s) d s \quad \text { for all }(z, x) \in \Omega \times \mathbb{R} .
$$

It is well-known that the critical points of the $C^{1}$-functional

$$
\Phi(u)=\frac{1}{p}\|D u\|_{p}^{p}+\frac{1}{p} \int_{\Omega} \beta(z)|u(z)|^{p} d z-\int_{\Omega} L(z, u(z)) d z, \quad u \in W^{1, p}(\Omega),
$$

are the weak solutions of problem $\left(\mathrm{P}_{1}\right)$.

The following proposition is a particular case of a more general result due to Motreanu and Papageoegiou [18].

Proposition 2.2. If $u_{0} \in W^{1, p}(\Omega)$ is a local $C^{1}(\bar{\Omega})$-minimizer of $\Phi$, i.e. there exists $\rho_{0}>0$ such that

$$
\Phi\left(u_{0}\right) \leq \Phi\left(u_{0}+h\right), \quad \text { for all } h \in C^{1}(\bar{\Omega}) \text { with }\|h\|_{C^{1}(\bar{\Omega})} \leq \rho_{0},
$$

then $u_{0} \in C^{1, \eta}(\bar{\Omega})$ with $\eta \in(0,1)$ and it is a local $W^{1, p}(\Omega)$-minimizer of $\Phi$, i.e. there exists $\rho_{1}>0$ such that

$$
\Phi\left(u_{0}\right) \leq \Phi\left(u_{0}+h\right), \quad \text { for all } h \in W^{1, p}(\Omega) \text { with }\|h\|_{W^{1, p}(\Omega)} \leq \rho_{1} .
$$

Let $x \in \mathbb{R}$. We set $x^{ \pm}:=\max \{ \pm x, 0\}$ and for $u \in W^{1, p}(\Omega)$, we define $u^{ \pm}(\cdot)=u(\cdot)^{ \pm}$. We know that $u^{ \pm} \in W^{1, p}(\Omega),|u|=u^{+}+u^{-}$and $u=u^{+}-u^{-}$.

The Nemytskii map corresponding to a measurable function $h: \Omega \times \mathbb{R} \rightarrow \mathbb{R}$ is indicated as

$$
N_{h}(u)(\cdot)=h(\cdot, u(\cdot)), \quad \text { for all } u \in W^{1, p}(\Omega) .
$$

Let $A: W^{1, p}(\Omega) \rightarrow W^{1, p}(\Omega)^{*}$ be the nonlinear map defined by

$$
\langle A(u), v\rangle=\int_{\Omega}\|D u\|^{p-2}(D u, D v)_{\mathbb{R}^{N}} d z, \quad u, v \in W^{1, p}(\Omega) .
$$

We have the following well-known result. 
Proposition 2.3. The nonlinear map A defined by (2.4) is maximal monotone, it maps bounded sets to bounded sets and it is of type $\left(\mathrm{S}_{+}\right)$, i.e. if $u_{n} \rightarrow u$ in $W^{1, p}(\Omega)$ and $\limsup _{n \rightarrow+\infty}\left\langle A\left(u_{n}\right), u_{n}-u\right\rangle \leq 0$, then $u_{n} \rightarrow u$ in $W^{1, p}(\Omega)$.

In [24] we find

LEMMA 2.4. Let $\beta \in L^{\infty}(\Omega)$ be a nonnegative and nontrivial function. Then, there exists $c_{0}>0$ such that

$$
\sigma(u):=\|D u\|_{p}^{p}+\int_{\Omega} \beta(z)|u(z)|^{p} d z \geq c_{0}\|u\|^{p}, \quad \text { for all } u \in W^{1, p}(\Omega) .
$$

Now, we introduce some suitable truncations associated with the nonlinearity $l$, which will play a crucial role in localizing the critical points of suitable energy functionals strictly related to the functional $\Phi$ associated to problem $\left(\mathrm{P}_{\lambda}\right)$.

Let $w$ and $v$ be two functions in $W^{1, p}(\Omega)$, with $v \leq w$, we consider the following three Carathéodory functions $l^{w}, l_{v}, l_{v}^{w}: \Omega \times \mathbb{R} \rightarrow \mathbb{R}$ defined, for every $(z, x) \in \Omega \times \mathbb{R}$, by putting

$$
\begin{aligned}
& l^{w}(z, x)=\left\{\begin{array}{ll}
l(z, x), & x \leq w(z), \\
l(z, w(z)), & x>w(z),
\end{array} \quad l_{v}(z, x)= \begin{cases}l(z, v(z)), & x<v(z) \\
l(z, x), & x \geq v(z)\end{cases} \right. \\
& l_{v}^{w}(z, x)= \begin{cases}l(z, v(z)), & x<v(z) \\
l(z, x), & v(z) \leq x \leq w(z), \\
l(z, w(z)), & x>w(z) .\end{cases}
\end{aligned}
$$

Moreover, denote with $L^{w}, L_{v}$ and $L_{v}^{w}$ the antiderivatives of $l^{w}, l_{v}$ and $l_{v}^{w}$ respectively (for instance, $L^{w}(z, x)=\int_{0}^{x} l^{w}(z, s) d s$ for every $(z, x) \in \Omega \times \mathbb{R}$ ). We consider the following functionals defined on $W^{1, p}(\Omega)$ :

$$
\begin{gathered}
\Phi^{w}(u)=\frac{\sigma(u)}{p}-\int_{\Omega} L^{w}(z, u(z)) d z, \quad \Phi_{v}(u)=\frac{\sigma(u)}{p}-\int_{\Omega} L_{v}(z, u(z)) d z \\
\Phi_{v}^{w}(u)=\frac{\sigma(u)}{p}-\int_{\Omega} L_{v}^{w}(z, u(z)) d z
\end{gathered}
$$

Such functionals, see [7], are weakly lower semicontinuous and continuously Gâteaux differentiable on $W^{1, p}(\Omega)$.

We give the following results which summarize some classical results for $\left(\mathrm{P}_{1}\right)$.

Lemma 2.5. Let $l: \Omega \times \mathbb{R} \rightarrow \mathbb{R}$ be a Carathéodory function.

$\left(\mathrm{l}_{1}\right)$ Assume that $\left(\mathrm{l}^{*}\right)$ holds with $r<p$. Then, problem $\left(\mathrm{P}_{1}\right)$ admits a weak solution $w$ which is a global minimum for the functional $\Phi$, i.e. $\Phi(w)=$ $\inf \left\{\Phi(u): u \in W^{1, p}(\Omega)\right\}$. In addition suppose that there exists $\theta \in \mathbb{R}$ such that

$$
\int_{\Omega}\left(\frac{\theta^{p}}{p} \beta(z)-L(z, \theta)\right) d z<0
$$

then $w$ is nontrivial. 
Let $w$ be a weak solution of problem $\left(\mathrm{P}_{1}\right)$. Then:

$\left(\mathrm{l}_{2}\right) w \in C^{1, \eta}(\bar{\Omega}), 0<\eta<1$.

$\left(1_{3}\right)$ Suppose that, for every $\rho>0$, there exists $\xi_{\rho} \geq 0$ such that the function $x l(z, x)+\xi_{\rho}|x|^{p} \geq 0$ for almost all $z \in \Omega$ provided that $|x| \leq \rho$. Then, if $w$ is nonnegative (nonpositive) and does not vanish identically in $\Omega$, $w \in \operatorname{int} C_{+}\left(-\operatorname{int} C_{+}\right)$.

$\left(\mathrm{l}_{4}\right)$ Assume that $l(z, 0) \geq 0$ for almost all $z \in \Omega$. Then every nontrivial weak solution of problem $\left(\mathrm{P}_{\mathrm{l}_{0}}\right)$ turns out to be a solution of problem $\left(\mathrm{P}_{1}\right)$, as well as, if $l(z, 0) \leq 0$ for almost all $z \in \Omega$, then every nontrivial weak solution of problem $\left(\mathrm{P}_{1^{0}}\right)$ turns out to be a solution of problem $\left(\mathrm{P}_{1}\right)$.

Proof. For completeness we give a sketch of the proof of the above assertions.

$\left(\mathrm{l}_{1}\right)$ Bearing in mind Lemma 2.4, because $W^{1, p}(\Omega)$ is embedded in $L^{r}(\Omega)$, for every $1<r<p^{*}$, there exist two positive constants $c_{1}$ and $c_{r}$ such that from the growth condition $\left(1^{*}\right)$, for every $u \in W^{1, p}(\Omega)$, we have

$\Phi(u) \geq c_{0}\|u\|^{p}-\|\alpha\|_{\infty}\|u\|_{1}-\frac{\|\alpha\|_{\infty}}{r}\|u\|_{r}^{r} \geq c_{0}\|u\|^{p}-c_{1}\|\alpha\|_{\infty}\|u\|-c_{r}^{r} \frac{\|\alpha\|_{\infty}}{r}\|u\|^{r}$,

which implies that $\Phi$ is coercive, since $r<p$. Moreover, standard arguments show that $\Phi$ is also weakly lower semicontinuous. Since, $W^{1, p}(\Omega)$ is a reflexive Banach space, the Weierstrass Theorem ensures that our conclusion holds. In particular, by (2.5), an immediate computation furnishes that $w$ is nontrivial.

$\left(\mathrm{l}_{2}\right)$ Taking into account [11], where the authors proved that $w \in L^{\infty}(\Omega)$, the assertion is an immediate consequence of the regularity results contained in [15].

$\left(l_{3}\right)$ Suppose that $w$ is a nonnegative and nontrivial solution of problem $\left(\mathrm{P}_{1}\right)$. Since, we have that $\Delta_{p} w \leq\left(\|\beta\|_{\infty}+\xi_{\rho}\right) w^{p-1}$, Vazquez's maximum principle [26] gives at once our conclusion. If $w$ is nonpositive, we can work analogously.

$\left(\mathrm{l}_{4}\right)$ If $w$ is a nontrivial weak solution of problem $\left(\mathrm{P}_{\mathrm{l}_{0}}\right)$, testing (2.1) with $v=-w^{-}$and by making use of Lemma 2.4, we have

$$
c_{0}\left\|w^{-}\right\|^{p} \leq \int_{\Omega}\left(\left\|D w^{-}\right\|^{p}+\beta(z)\left|w^{-}\right|^{p}\right) d z=-\int_{\Omega} l(z, 0) w^{-} d z \leq 0
$$

hence $w \geq 0$ and therefore $w$ is also a solution of problem $\left(\mathrm{P}_{1}\right)$. In the other case, we work in a similar way.

LEMma 2.6. Let $\underline{w}$ and $\bar{w}$ be respectively a sub-solution and a super-solution of problem $\left(\mathrm{P}_{1}\right)$ such that $\underline{w} \leq \bar{w}$, we have:

(a) If $w$ is a critical point of $\Phi^{\bar{w}}$, then one has that $w \leq \bar{w}$.

(b) If $w$ is a critical point of $\Phi_{\underline{w}}$, then one has that $\underline{w} \leq w$.

(c) If $w$ is a critical point of $\Phi_{\underline{w}}^{\bar{w}}$, then one has that $\underline{w} \leq w \leq \bar{w}$. 
Proof. We give the proof just in the first case, because in the others cases we can work in a similar way. Since, $w$ is a critical point of $\Phi^{\bar{w}}$, we have

$$
A(w)+\beta|w|^{p-1} w=N_{l^{\bar{w}}}(w) .
$$

We act on this with $(w-\bar{w})^{+} \in W^{1, p}(\Omega)$ and bearing in mind that $\bar{w}$ is a super-solution for problem $\left(\mathrm{P}_{1}\right)$, we get

$$
\begin{aligned}
& \left\langle A(w),(w-\bar{w})^{+}\right\rangle+\int_{\Omega} \beta|w|^{p-2} w(w-\bar{w})^{+} d z=\int_{\Omega} l^{\bar{w}}(z, w(z))(w-\bar{w})^{+} d z \\
= & \int_{\Omega} l^{\bar{w}}(z, \bar{w}(z))(w-\bar{w})^{+} d z \leq\left\langle A(\bar{w}),(w-\bar{w})^{+}\right\rangle+\int_{\Omega} \beta|\bar{w}|^{p-2} \bar{w}(w-\bar{w})^{+} d z .
\end{aligned}
$$

From this, we infer that

$$
\begin{aligned}
\int_{\{w>\bar{w}\}}\left(|| D w \|^{p-2} D w-\right. & \left.\|D \bar{w}\|^{p-2} D \bar{w}, D w-D \bar{w}\right)_{\mathbb{R}^{N}} d z \\
& +\int_{\{w>\bar{w}\}} \beta\left(|w|^{p-2} w-|\bar{w}|^{p-2} \bar{w}\right)(w-\bar{w})^{+} d z \leq 0 .
\end{aligned}
$$

Hence, since $\beta \geq 0$, according to $2 \leq p$ or $1<p<2$ there exist two positive constants $b_{1}$ and $b_{2}$, see Gasinski and Papageorgiou [7, p. 740], such that, for $i=1,2$, we have

$$
b_{i}\left\|D(w-\bar{w})^{+}\right\|_{p}^{p}+\int_{\Omega} \beta\left((w-\bar{w})^{+}\right)^{p} d z \leq 0
$$

which clearly implies that $|\{w>\bar{w}\}|_{N}=0$, i.e. $w \leq \bar{w}$.

REMARK 2.7. We observe that if $l(z, 0) \geq 0$ for almost all $z \in \Omega$, we have that $w=0$ is a sub-solution of problem $\left(\mathrm{P}_{1}\right)$ as well as if $l(z, 0) \leq 0$ for almost all $z \in \Omega$, we have that $w=0$ is a super-solution of problem $\left(\mathrm{P}_{1}\right)$. So, it is clear that the conclusions of Lemma 2.5 in case $\left(l_{4}\right)$ can be obtained also from (a) and (b) of Lemma 2.6.

Now, we consider the following nonlinear eigenvalue problem:

$$
\begin{cases}-\Delta_{p} u+\beta(z)|u|^{p-2} u=\lambda|u|^{p-2} u & \text { in } \Omega \\ \frac{\partial u}{\partial n}=0 & \text { on } \Omega\end{cases}
$$

with $\beta \in L^{\infty}(\Omega)$, nonnegative and nontrivial. A more general version of (2.6) is studied in [22], where the potential function $\beta(\cdot)$ is indefinite (i.e. sign changing). So, in [22], it is proved that problem $(2.6)$ has a smallest eigenvalue $\hat{\lambda}_{1}(\beta)$. In our case, for this eigenvalue, we have:

- $\widehat{\lambda}_{1}(\beta)>0$, see Lemma 2.4 ;

- $\widehat{\lambda}_{1}(\beta)$ is simple (i.e. if $u, v$ are two eigenfunctions corresponding to $\widehat{\lambda}_{1}(\beta)$, then $u=\xi v$ for some $\xi \neq 0$ ); 
- $\widehat{\lambda}_{1}(\beta)$ is isolated (i.e. there exists $\varepsilon>0$ such that $\left(\widehat{\lambda}_{1}(\beta), \widehat{\lambda}_{1}(\beta)+\varepsilon\right)$ does not contain any eigenvalue of $\left(-\Delta_{p}, W^{1, p}(\Omega), \beta\right)$.

The eigenvalue has the following variational characterization:

$$
\widehat{\lambda}_{1}(\beta)=\inf \left\{\frac{\sigma(u)}{\|u\|_{p}^{p}}: u \in W^{1, p}(\Omega), u \neq 0\right\},
$$

where $\sigma(u)$ has been introduced in Lemma 2.4. The infimum is actually realized on the one dimensional eigenspace corresponding to $\widehat{\lambda}_{1}(\beta)>0$. It is clear from (2.7) that the elements of this eigenspace do not change sign. By $\widehat{u}_{1}$ we denote the positive, $L^{p}$-normalized (i.e. $\left\|\widehat{u}_{1}\right\|_{p}=1$ ) eigenfunction corresponding to $\widehat{\lambda}_{1}(\beta)>$ 0 . Lemma 2.5 implies that $\widehat{u}_{1} \in \operatorname{int} C_{+}$.

Using the isolation of $\hat{\lambda}_{1}(\beta)>0$, we can also define the second eigenvalue $\widehat{\lambda}_{2}(\beta)>0$ and via the Lusternik-Schnirelmann minimax scheme, we can have a whole sequence $\left\{\widehat{\lambda}_{k}(\beta)\right\}_{k \geq 1}$ of distinct eigenvalues (usually called "variational eigenvalues") such that $\widehat{\lambda}_{k}(\beta) \uparrow+\infty$. We do not know if these are all eigenvalues. This is the case, if $N=1$ (ordinary differential equations) or $p=2$ (linear eigenvalue problem).

In this latter case, by $E\left(\widehat{\lambda}_{k}(\beta)\right)$, we denote the eigenspace corresponding to $\widehat{\lambda}_{k}(\beta)$. As a direct consequence of this property, we have that

$\left(\theta_{1}\right)$ If $\Theta(z) \geq \widehat{\lambda}_{k}(\beta)$ for almost all $z \in \Omega, \Theta(z) \neq \widehat{\lambda}_{k}(\beta)$ with $k \geq 1$, then

$$
\sigma(u)-\int_{\Omega} \Theta u^{2} d z \leq-\varepsilon_{1}\|u\|^{2} \quad \text { for all } u \in \bigoplus_{i=1}^{k} E\left(\widehat{\lambda}_{i}(\beta)\right) \text {, and some } \varepsilon_{1}>0 \text {. }
$$

$\left(\theta_{2}\right)$ If $\Theta(z) \leq \widehat{\lambda}_{k+1}(\beta)$ for almost all $z \in \Omega, \Theta(z) \neq \widehat{\lambda}_{k}(\beta)$ with $k \geq 1$, then

$$
\sigma(u)-\int_{\Omega} \Theta u^{2} d z \geq \varepsilon_{2}\|u\|^{2} \quad \text { for all } u \in \overline{\bigoplus_{i \geq k+1} E\left(\widehat{\lambda}_{i}(\beta)\right)} \text { and some } \varepsilon_{2}>0 \text {. }
$$

Next let us recall some basic facts from Morse theory. Let $X$ be a Banach space and let $\left(Y_{1}, Y_{2}\right)$ be a topological pair such that $Y_{2} \subseteq Y_{1} \subseteq X$. For every integer $k \geq 0$, by $H_{k}\left(Y_{1}, Y_{2}\right)$ we denote the $k^{\text {th }}$ relative singular homology group with integer coefficients. For $k<0$, we have $H_{k}\left(Y_{1}, Y_{2}\right)=0$.

Given $\varphi \in C^{1}(X)$ and $c \in \mathbb{R}$, we introduce the following sets:

$$
\begin{gathered}
\varphi^{c}:=\{x \in X: \varphi(x) \leq c\}, \\
K_{\varphi}:=\left\{x \in X: \varphi^{\prime}(x)=0\right\}, \quad K_{\varphi}^{c}:=\left\{x \in K_{\varphi}: \varphi(x)=c\right\} .
\end{gathered}
$$

Let $u \in X$ be an isolated critical point of $\varphi$ with $\varphi(x)=c$ (i.e. $\left.u \in K_{\varphi}^{c}\right)$. The critical groups of $\varphi$ at $u$ are defined by

$$
C_{k}(\varphi, u)=H_{k}\left(\varphi^{c} \cap U, \varphi^{c} \cap U \backslash\{u\}\right), \quad \text { for all } k \geq 0,
$$

where $U$ is a neighbourhood of $u$ such that $K_{\varphi} \cap \varphi^{c} \cap U=\{u\}$. The excision property of singular homology implies that this definition of critical groups is independent of the particular choice of the neighbourhood $U$. 
Suppose that $\varphi \in C^{1}(X)$ satisfies the "C-condition" and $\inf \varphi\left(K_{\varphi}\right)>-\infty$. Let $c<\inf \varphi\left(K_{\varphi}\right)$. The critical groups of $\varphi$ at infinity are defined by

$$
C_{k}(\varphi, \infty)=H_{k}\left(X, \varphi^{c}\right), \quad \text { for all } k \geq 0 .
$$

By virtue of the second deformation theorem (see, for example, Gasinski-Papageorgiou [7, p. 628]), we have that the above definition of critical groups at infinity is independent of the choice of the level $c<\inf \varphi\left(K_{\varphi}\right)$.

\section{Solutions of constant sign}

In this section, we produce nontrivial solutions of constant sign and we localize them. For this purpose, we introduce the following conditions on the data of $\left(\mathrm{P}_{\lambda}\right)$.

$\mathrm{H}(\beta) \beta \in L^{\infty}(\Omega)$ is a nonnegative and nontrivial function.

$\left(\mathrm{H}_{1}\right)$ Let $f: \Omega \times \mathbb{R} \rightarrow \mathbb{R}$ be a Carathéodory function such that $f(z, 0)=0$ for almost all $z \in \Omega$ and satisfying:

(a) for every $\rho>0$, there exists $a_{\rho} \in L^{\infty}(\Omega)$ such that $|f(z, x)| \leq a_{\rho}(z)$ for almost all $z \in \Omega$ and all $|x| \leq \rho$;

(b) there exist $\eta \in L^{\infty}(\Omega)$ and a positive constant $\widehat{\eta}$ such that $\eta(z) \geq$ $\widehat{\lambda}_{1}(\beta)$ almost everywhere in $\Omega$ and

$\eta(z) \leq \liminf _{x \rightarrow \pm \infty} \frac{f(z, x)}{|x|^{p-2} x} \leq \limsup _{x \rightarrow \pm \infty} \frac{f(z, x)}{|x|^{p-2} x} \leq \widehat{\eta} \quad$ uniformly for a.a. $z \in \Omega ;$

(c) there exist $\xi_{0}>0$ and $\tau \in(q, p]$ such that

$\xi_{0} \leq \liminf _{x \rightarrow \pm \infty} \frac{x f(z, x)-p F(z, x)}{|x|^{\tau}}$ uniformly for a.a. $z \in \Omega$,

with $F(z, x)=\int_{0}^{x} f(z, s) d s,(z, x) \in \Omega \times \mathbb{R}$

(d) there exist $\delta_{0} \in(0,1)$ and $r>p$ such that

$0 \leq x f(z, x) \leq c_{1}|x|^{r}, \quad$ for a.a. $z \in \Omega$ and $|x| \leq \delta_{0}$, and some $c_{1}>0$,

and for every $\mu \in\left(0, \delta_{0}\right)$, we can find $\varepsilon_{\mu}>0$ such that

$\varepsilon_{\mu} \leq|f(z, x)|, \quad$ for a.a. $z \in \Omega$ and all $x \in\left[\mu, \delta_{0}\right] ;$

(e) for every $\rho>0$, there exists $\xi_{\rho}>0$ such that for almost all $z \in \Omega$, the function

$$
x \mapsto f(z, x)+\xi_{\rho}|x|^{p-2} x \in \mathbb{R},
$$

is nondecreasing on $[-\rho, \rho]$.

Just to show that the class of functions satisfying $\mathrm{H}(\beta)$ and $\left(\mathrm{H}_{1}\right)$ is nonempty, we give 
EXAMPLE 3.1. Let $\alpha \in C^{0}(\Omega)$ with $\inf _{z \in \Omega} \alpha(z) \geq \alpha_{0}>0$ and let $\mu, \eta, r, c_{1}$ and $c_{2}$ be five positive constants such that

$$
q<\eta<p<r, \quad c_{2}-c_{1}=1, \quad c_{2} \alpha_{0}>\widehat{\lambda}_{1}(\beta) .
$$

The following function, defined in $\Omega \times \mathbb{R}$, satisfies hypotheses $\left(\mathrm{H}_{\beta}\right)$ and $\left(\mathrm{H}_{1}\right)$ :

$$
f(z, x)= \begin{cases}\alpha(z)|x|^{r-2} x, & |x| \leq 1 \\ \alpha(z)\left(c_{2}|x|^{p-2} x-c_{1}|x|^{\eta-2} x\right), & |x|>1\end{cases}
$$

Now, to study problem $\left(\mathrm{P}_{\lambda}\right)$, for every $\lambda>0$, we consider the following Carathéodory function $l_{\lambda}: \Omega \times \mathbb{R} \rightarrow \mathbb{R}$ defined by

$$
l_{\lambda}(z, x)=\lambda|x|^{q-2} x+f(z, x), \quad(z, x) \in \Omega \times \mathbb{R},
$$

and the corresponding energy functional $\Phi_{\lambda}: W^{1, p}(\Omega) \rightarrow \mathbb{R}$ given by

$$
\Phi_{\lambda}(u)=\frac{\sigma(u)}{p}-\int_{\Omega} L_{\lambda}(z, u(z)) d z, \quad \text { for all } u \in W^{1, p}(\Omega),
$$

where $\sigma(u)$ is given in Lemma 2.4 and $L_{\lambda}(z, x)=\int_{0}^{x} l_{\lambda}(z, s) d s$ for $(z, x) \in$ $\Omega \times \mathbb{R}$. Evidently $\Phi_{\lambda} \in C^{1}\left(W^{1, p}(\Omega)\right)$. Hypothesis $\left(\mathrm{H}_{1}\right)(\mathrm{b})$ makes $\Phi_{\lambda}$ indefinite (noncoercive).

Proposition 3.2. If hypotheses $\mathrm{H}(\beta)$ and $\left(\mathrm{H}_{1}\right)(\mathrm{d})$, (e) hold, then there exists $\lambda^{*}>0$ such that for every $\lambda \in\left(0, \lambda^{*}\right)$ problem $\left(\mathrm{P}_{\lambda}\right)$ has two nontrivial constant sign solutions $u_{0} \in \operatorname{int} C_{+}$and $v_{0} \in-\operatorname{int} C_{+}$, both local minimizers of the functional $\Phi_{\lambda}$.

Proof. Let $\widehat{u}_{1} \in \operatorname{int} C_{+}$be the positive $L^{p}$-normalized principal eigenfunction for problem (2.6). Since $1<q<p$ and $\delta_{0} \in(0,1)$ is as in $\left(\mathrm{H}_{1}\right)(\mathrm{d})$, there exists $t \in(0,1)$, small enough, such that

$$
t \widehat{u}_{1}(z) \in\left(0, \delta_{0}\right], \quad\left(\widehat{\lambda}_{1}(\beta)+\|\beta\|_{\infty}\right)\left(t \widehat{u}_{1}^{p-1}(z)\right) \leq \lambda\left(t \widehat{u}_{1}^{q-1}(z)\right)
$$

for all $z \in \bar{\Omega}$. From this, owing to $\left(\mathrm{H}_{1}\right)(\mathrm{d})$, we prove that $\underline{u}:=t \widehat{u}_{1} \in \operatorname{int} C_{+}$is a sub-solution of problem $\left(\mathrm{P}_{\lambda}\right)$. Indeed, one has

$(3.2)-\Delta_{p} \underline{u}(z)+\beta(z) \underline{u}^{p-1}(z) \leq\left(\widehat{\lambda}_{1}(\beta)+\|\beta\|_{\infty}\right) \underline{u}^{p-1} \leq \lambda \underline{u}^{q-1}(z)+f(z, \underline{u}(z))$.

To construct a super-solution for problem $\left(\mathrm{P}_{\lambda}\right)$, we use the following auxiliary Neumann problem:

$$
-\Delta_{p} c+\beta(z) c^{p-1}=1 \quad \text { in } \Omega, \quad \frac{\partial c}{\partial n}=0 \quad \text { on } \partial \Omega, c>0,
$$

which, by Lemma 2.5, admits a solution $c \in \operatorname{int} C_{+}$.

Claim. There exists $\lambda^{*}>0$ such that for every $\lambda \in\left(0, \lambda^{*}\right)$ we can find $\eta_{1}=\eta_{1}(\lambda) \in\left(0, \delta_{0} /\|c\|_{\infty}\right)$ such that

$$
\lambda\left(\eta_{1}\|c\|_{\infty}\right)^{q-1}+c_{1}\left(\eta_{1}\|c\|_{\infty}\right)^{r-1}<\eta_{1}^{p-1},
$$


where $c_{1}$ is involved in $\left(\mathrm{H}_{1}\right)(\mathrm{d})$. We argue by contradiction. So, suppose that the claim is not true. Then, we can find $\lambda_{n} \rightarrow 0^{+}$such that, for every $\eta \in$ $\left(0, \delta_{0} /\|c\|_{\infty}\right)$, we have

$$
\eta^{p-1} \leq \lambda_{n}\left(\eta\|c\|_{\infty}\right)^{q-1}+c_{1}\left(\eta\|c\|_{\infty}\right)^{r-1} .
$$

From this, when $n$ tends to $+\infty$, we get $\eta^{p-1} \leq c_{1}\left(\eta\|c\|_{\infty}\right)^{r-1}$, that is $1 \leq$ $c_{1} \eta^{r-p}\|c\|_{\infty}^{r-1}$. Since $r>p$ and $\eta \in\left(0, \delta_{0} /\|c\|_{\infty}\right)$ is arbitrary, when $\eta \rightarrow 0^{+}$, we reach a contradiction and this proves the claim.

Let $\bar{u}=\eta_{1} c \in \operatorname{int} C_{+}$. We observe that $\bar{u}(z)=\eta_{1} c(z) \leq \delta_{0}$ for all $z \in \Omega$ and by making use of $(3.3)$ and $\left(\mathrm{H}_{1}\right)(\mathrm{d})$, for almost all $z \in \Omega$, we obtain

$$
\begin{aligned}
-\Delta_{p} \bar{u}(z)+\beta(z) \bar{u}^{p-1} & =\eta_{1}^{p-1}\left[A(c)+\beta c^{p-1}\right]=\eta_{1}^{p-1} \\
& >\lambda \bar{u}^{q-1}(z)+c_{1} \bar{u}^{r-1} \geq \lambda \bar{u}^{q-1}(z)+f(z, \bar{u}(z)),
\end{aligned}
$$

that is, $\bar{u}$ is a super-solution of problem $\left(\mathrm{P}_{\lambda}\right)$. Since $\bar{u} \in \operatorname{int} C_{+}$, by taking $t \in(0,1)$ even smaller, we can have $\underline{u}=t \widehat{u}_{1} \leq \bar{u}$.

We consider the following truncation of the reaction term of $\left(\mathrm{P}_{\lambda}\right)$ (see Lemma 2.5):

$$
\left(l_{\lambda}\right)_{\underline{u}}^{\bar{u}}(z, x):= \begin{cases}l_{\lambda}(z, \underline{u}(z)), & x<\underline{u}(z), \\ l_{\lambda}(z, x), & \underline{u}(z) \leq x \leq \bar{u}(z), \\ l_{\lambda}(z, \bar{u}(z)), & x>\bar{u}(z),\end{cases}
$$

where $l_{\lambda}(z, x)=\lambda|x|^{q-2} x+f(z, x)$ with $(z, x) \in \Omega \times \mathbb{R}$ and the corresponding $C^{1}$-functional, namely

$$
\left(\Phi_{\lambda}\right)_{\underline{u}}^{\bar{u}}(u)=\frac{\sigma(u)}{p}-\int_{\Omega}\left(L_{\lambda}\right)_{\underline{u}}^{\bar{u}}(z, u(z)) d z, \quad \text { for all } u \in W^{1, p}(\Omega) .
$$

Using Lemmas 2.5 and 2.6, the functional $\left(\Phi_{\lambda}\right)_{\underline{u}}^{\bar{u}}$ admits a critical point $u_{0} \in$ $\operatorname{int} C_{+}$which is a global minimum such that $\underline{u} \leq u_{0} \leq \bar{u}$. More precisely, we can improve this conclusion, we prove that

$$
u_{0} \in \operatorname{int}_{C^{1}(\bar{\Omega})}[\underline{u}, \bar{u}] .
$$

Indeed, let $\delta \in\left(0, \min _{\Omega} u_{0}\right)$ and set $u_{\delta}=u_{0}-\delta \in \operatorname{int} C_{+}$. Let $\rho=\|\bar{u}\|_{\infty}$ and let $\xi_{\rho}>0$ be as postulated in $\left(\mathrm{H}_{1}\right)(\mathrm{e})$. We have that there exists $\theta(\delta) \rightarrow 0^{+}$as $\delta \rightarrow 0^{+}$, such that, by $(3.1)$, we get

$$
\begin{aligned}
-\Delta_{p} u_{\delta}(z) & +\left(\beta(z)+\xi_{\rho}\right) u_{\delta}^{p-1}(z) \\
\geq & -\Delta_{p} u_{0}(z)+\left(\beta(z)+\xi_{\rho}\right) u_{0}^{p-1}(z)-\theta(\delta) \\
= & \lambda u_{0}^{q-1}(z)+f\left(z, u_{0}(z)\right)+\xi_{\rho} u_{0}^{p-1}(z)-\theta(\delta) \\
\geq & \lambda \underline{u}^{q-1}(z)(z)+f\left(z, \underline{u}^{-1}(z)\right)+\xi_{\rho} \underline{u}^{p-1}(z)-\theta(\delta) \\
\geq & -\Delta_{p} \underline{u}(z)+\beta(z) \underline{u}^{p-1}(z)+\xi_{\rho} \underline{u}^{p-1}(z)+f(z, \underline{u}(z))-\theta(\delta) .
\end{aligned}
$$


Let $\mu=\min _{\bar{\Omega}} \underline{u}>0$, with $\underline{u} \in \operatorname{int} C_{+}$. Then by virtue of hypothesis $\left(\mathrm{H}_{1}\right)(\mathrm{d})$, we have $f(z, \underline{u}(z)) \geq \xi_{\mu}>0$, for almost all $z \in \Omega$. Thus, since $\theta(\delta) \rightarrow 0^{+}$as $\delta \rightarrow 0^{+}$, we can find $\delta^{*}$ such that, for all $\delta \in\left(0, \delta^{*}\right]$, we have

$$
f(z, \underline{u}(z)) \geq \xi_{\mu} \geq \theta(\delta), \quad \text { for a.a. } z \in \Omega .
$$

Hence from (3.6), we have

$$
-\Delta_{p} u_{\delta}(z)+\left(\beta(z)+\xi_{\rho}\right) u_{\delta}^{p-1}(z) \geq-\Delta_{p} \underline{u}(z)+\left(\beta(z)+\xi_{\rho}\right) \underline{u}^{p-1}(z) \quad \text { a.e. in } \Omega,
$$

which implies that

$$
\underline{u} \leq u_{\delta} \Rightarrow u_{0}-, \quad \underline{u} \in \operatorname{int} C_{+} .
$$

Similarly, taking into account (3.3) and (3.4), if for $\delta>0$ we set $\widehat{u}_{\delta}=u_{\delta}+\delta \in$ $\operatorname{int} C_{+}$, then

$$
\begin{array}{rlrl}
-\Delta_{p} \widehat{u}_{\delta}(z) & +\beta(z) \widehat{u}_{\delta}^{p-1}(z) & \\
\leq & -\Delta_{p} u_{0}(z)+\beta(z) u_{0}^{p-1}(z)+\widehat{\rho}(\delta) & & \text { with } \widehat{\rho}(\delta) \text { as } \delta \rightarrow 0^{+}, \\
= & \lambda u_{0}^{q-1}(z)+f\left(z, u_{0}(z)\right)+\widehat{\rho}(\delta) & \\
\leq & \lambda u_{0}^{q-1}(z)+c_{1} u_{0}^{p-1}(z)+\widehat{\rho}(\delta) & \\
\leq & \lambda\left(\eta_{1}\|c\|_{\infty}\right)^{q-1}+c_{1}\left(\eta_{1}\|c\|_{\infty}\right)^{r-1}+\widehat{\rho}(\delta) & \\
\leq & & \\
\leq & & & \text { for all } \delta \in\left(0, \delta^{*}\right), \\
= & & \text { for some } \delta^{*}>0,
\end{array}
$$

Hence, arguing in a similar way as in the proof of Lemma 2.6, it follows that $\widehat{u}_{\delta} \leq \bar{u}$ which ensures that

$$
\bar{u}-u_{0} \in \operatorname{int} C_{+} .
$$

From (3.7) and (3.8), we have $u_{0} \in \operatorname{int}_{C^{1}(\bar{\Omega})}[\underline{u}, \bar{u}]$ and it follows that

$$
\left.\Phi_{\lambda}\right|_{[\underline{u}, \bar{u}]}=\left.\left(\Phi_{\lambda}\right)_{\underline{u}}^{\bar{u}}\right|_{[\underline{u}, \bar{u}]}+d, \quad \text { for some } d \in \mathbb{R}_{+} .
$$

Therefore, we have that $u_{0}$ is a local $C^{1}(\bar{\Omega})$-minimizer of $\Phi_{\lambda}$ and thanks to Proposition 2.2, a local $W^{1, p}(\Omega)$-minimizer of $\Phi_{\lambda}$.

Working with $\left(l_{\lambda}\right)_{v}^{\bar{v}}(z, x)$, we can produce as above a nontrivial negative solution $v_{0} \in-\operatorname{int} C_{+}$with $v_{0} \in \operatorname{int}_{C^{1}(\bar{\Omega})}[\underline{v}, \bar{v}]$, where $\underline{v}=-\eta_{1} c$ and $\bar{v}=-t \widehat{u}_{1}$ with $t \in(0,1)$ small. Moreover, $v_{0}$ is a local minimizer of $\Phi_{\lambda}$.

To produce extremal constant sign solutions for problem $\left(\mathrm{P}_{\lambda}\right)$ for every $\lambda \in\left(0, \lambda^{*}\right)$, i.e. a smallest nontrivial positive solution and a biggest nontrivial negative solution, we consider the following auxiliary problem:

$$
-\Delta_{p} u+\beta(z)|u|^{p-2} u=\lambda|u|^{q-2} u \quad \text { in } \Omega, \quad \frac{\partial u}{\partial n}=0 \quad \text { on } \partial \Omega .
$$


For this problem we have the following result.

Proposition 3.3. If hypothesis $\mathrm{H}(\beta)$ holds and $\lambda>0$, then problem (3.9) admits a unique positive solution $\widehat{u}_{\lambda} \in \operatorname{int} C_{+}$and a unique negative solution $\widehat{v}_{\lambda}=-\widehat{u}_{\lambda} \in-\operatorname{int} C_{+}$.

Proof. Since $q<p$, the existence of a nontrivial solution $\widehat{u}_{\lambda} \in \operatorname{int} C_{+}$is an immediate consequence of Lemma 2.5. To prove the uniqueness, we consider the integral functional $\gamma_{+}: L^{1}(\Omega) \rightarrow \overline{\mathbb{R}}=\mathbb{R} \cup\{+\infty\}$ defined by

$$
\gamma_{+}(u):= \begin{cases}\frac{1}{p}\left\|D u^{1 / p}\right\|_{p}^{p}+\frac{1}{p} \int_{\Omega} \beta(z) u(z) d z & \text { for } u \geq 0, u^{1 / p} \in W^{1, p}(\Omega), \\ +\infty & \text { otherwise. }\end{cases}
$$

From Diaz-Saá [5, Lemma 1], we know that $\gamma_{+}$is convex. Also, by Fatou's Lemma, $\gamma_{+}$is lower semicontinuos.

Let $u, v \in \operatorname{int} C_{+}$be two nontrivial positive solutions of (3.9). Then $u, v \in$ $\operatorname{dom} \gamma_{+}$and if $h \in C^{1}(\bar{\Omega})$ for $t \in(-1,1)$ small in absolute value, we have that $u+t h$ and $v+t h \in \operatorname{dom} \gamma_{+}$and so $\gamma_{+}$is Gâteaux differentiable at $u$ and $v$ in the direction $h$. In fact, using the chain rule and the density of $C^{1}(\bar{\Omega})$ in $W^{1, p}(\Omega)$, for every $h \in W^{1, p}(\Omega)$ we have

$\gamma_{+}^{\prime}\left(u^{p}\right)(h)=\frac{1}{p} \int_{\Omega} \frac{-\Delta_{p} u+\beta u^{p-1}}{u^{p-1}} h d z, \quad \gamma_{+}^{\prime}\left(v^{p}\right)(h)=\frac{1}{p} \int_{\Omega} \frac{-\Delta_{p} v+\beta v^{p-1}}{v^{p-1}} h d z$.

By virtue of the convexity of $\gamma_{+}$, we have that the map $u \mapsto \gamma_{+}^{\prime}(u)$ is monotone and so it follows

$$
\begin{aligned}
0 & \leq p\left\langle\gamma_{+}^{\prime}\left(u^{p}\right)-\gamma_{+}^{\prime}\left(v^{p}\right), u^{p}-v^{p}\right\rangle_{L^{1}(\Omega)} \\
& =\int_{\Omega}\left(\frac{-\Delta_{p} u+\beta u^{p-1}}{u^{p-1}}-\frac{-\Delta_{p} v+\beta v^{p-1}}{v^{p-1}}\right)\left(u^{p}-v^{p}\right) d z \\
& =\lambda \int_{\Omega}\left(\frac{1}{u^{p-q}}-\frac{1}{v^{p-q}}\right)\left(u^{p}-v^{p}\right) d z \leq 0
\end{aligned}
$$

which implies that $u=v$. This proves the uniqueness of the solution $\widehat{u}_{\lambda} \in \operatorname{int} C_{+}$. Since the nonlinearity $|u(\cdot)|^{q-2} u(\cdot)$ is odd, $\widehat{v}_{\lambda}=-\widehat{u}_{\lambda} \in-\operatorname{int} C_{+}$is the unique negative solution of problem $(3.9)$.

Another auxiliary result that we will need to reach our goal of establishing the existence of extremal nontrivial constant sign solutions for problem $\left(\mathrm{P}_{\lambda}\right)$ for every $\lambda \in\left(0, \lambda^{*}\right)$ is the following:

Proposition 3.4. If hypotheses $\mathrm{H}(\beta)$ and $\left(\mathrm{H}_{1}\right)(\mathrm{d})$, (e) hold, $\lambda \in\left(0, \lambda^{*}\right)$ and $t \in(0,1)$, then problem $\left(\mathrm{P}_{\lambda}\right)$ has a smallest solution in $[$ tu, $\bar{u}]$ and a biggest solution in $[\underline{v}, t \bar{v}]$.

ProOF. Let $\widehat{S}_{\lambda}^{+}$be the set of solutions of $\left(\mathrm{P}_{\lambda}\right)$ in the order interval $[t \underline{u}, \bar{u}]$ (see the proof of Proposition 3.2). From Aizicovici, Papageorgiou and Staicu [1], 
we know that the set $\widehat{S}_{\lambda}^{+}$is downward directed (i.e. if $u_{1}, u_{2} \in \widehat{S}_{\lambda}^{+}$, there exists $u \in \widehat{S}_{\lambda}^{+}$such that $u \leq u_{1}$ and $u \leq u_{2}$ ). Let $C \subseteq \widehat{S}_{\lambda}^{+}$be a chain (i.e. a totally ordered subset of $\widehat{S}_{\lambda}^{+}$). From Dunford and Schwartz [6], we can find a sequence $\left\{u_{n}\right\}_{n \geq 1} \subseteq C$ such that $\inf C=\inf _{n \geq 1} u_{n}$. Clearly, for every $n \geq 1$, we have

$$
A\left(u_{n}\right)+\beta u_{n}^{p-1}=\lambda u_{n}^{q-1}+N_{f}\left(u_{n}\right), \quad u_{n} \in[\underline{t}, \bar{u}] .
$$

Consequently, it is a simple matter to see that the sequence $\left\{u_{n}\right\}$ is bounded in $W^{1, p}(\Omega)$. So, we may assume that

$$
u_{n} \rightarrow u \quad \text { in } W^{1, p}(\Omega) \quad \text { and } \quad u_{n} \rightarrow u \quad \text { in } L^{p}(\Omega) \text { as } n \rightarrow \infty .
$$

On (3.10) we act with $u_{n}-u \in W^{1, p}(\Omega)$, pass to the limit as $n \rightarrow \infty$ and use (3.11) to prove that

$$
\lim _{n \rightarrow \infty}\left\langle A\left(u_{n}\right), u_{n}-u\right\rangle=0,
$$

which implies, see Proposition 2.3,

$$
u_{n} \rightarrow u \in W^{1, p}(\Omega) \text {. }
$$

Therefore, if in (3.10) we pass to the limit as $n \rightarrow \infty$ and use (3.12), then we have

$$
A(u)+\beta u^{p-1}=\lambda u^{q-1}+N_{f}(u),
$$

that is $u \in \widehat{S}_{\lambda}^{+}$and $u=\inf C$. Since $C$ is an arbitrary chain in $\widehat{S}_{\lambda}^{+}$, by virtue of the Kuratowski-Zorn Lemma, we have that there exists a minimal element $\widehat{u}_{0}$ of $\widehat{S}_{\lambda}^{+}$. Let $v \in \widehat{S}_{\lambda}^{+}$. Since $\widehat{S}_{\lambda}^{+}$is downward directed, we can find $y \in \widehat{S}_{\lambda}^{+}$such that $y \leq \widehat{u}_{0}$ and $y \leq v$. The minimality of $\widehat{u}_{0}$ implies that $\widehat{u}_{0}=y$ and $\widehat{u}_{0} \leq v$, hence $\widehat{u}_{0}$ is the smallest element of $\widehat{S}_{\lambda}^{+}$.

Let $\widehat{S}_{\lambda}^{-}$be the set of solutions in the order interval $[\underline{v}, t \bar{v}]$. This set is upward directed (i.e. if $v_{1}, v_{2} \in \widehat{S}_{\lambda}^{-}$, there exists $v \in \widehat{S}_{\lambda}^{-}$such that $v \geq v_{1}$ and $v \geq v_{2}$, see [1]). Reasoning as above, via the Kuratowski-Zorn Lemma, we produce $\widehat{v}_{0} \in-\operatorname{int} C_{+}$, the biggest element of $\widehat{S}_{\lambda}^{-}$.

Now, we are ready to produce the extremal nontrivial constant sign solutions for problem $\left(\mathrm{P}_{\lambda}\right)$ for every $\lambda \in\left(0, \lambda^{*}\right)$.

Proposition 3.5. If hypotheses $\mathrm{H}(\beta)$ and $\mathrm{H}_{1}(\mathrm{~d})$, (e) hold and $\lambda \in\left(0, \lambda^{*}\right)$, then problem $\left(\mathrm{P}_{\lambda}\right)$ has a smallest positive solution $u_{*} \in \operatorname{int} C_{+}$and a biggest negative solution $v_{*} \in-\operatorname{int} C_{+}$.

Proof. Let $\varepsilon_{n} \in(0,1), \varepsilon_{n} \downarrow 0^{+}$. For all $n \geq 1$, from Proposition 3.4 we know that problem $\left(\mathrm{P}_{\lambda}\right)$ has a smallest solution $\widehat{u}_{n} \in\left[\underline{u}_{n}=\varepsilon_{n} \underline{u}, \bar{u}\right]$. Moreover, we have

$$
\begin{aligned}
-\Delta_{p} \widehat{u}_{n}(z)+\beta(z) \widehat{u}_{n}^{p-1}(z) & =\lambda \widehat{u}_{n}^{q-1}(z)+f\left(z, \widehat{u}_{n}(z)\right) & & \text { a.a. } z \in \Omega, \\
\frac{\partial \widehat{u}_{n}}{\partial n} & =0 & & \text { on } \partial \Omega .
\end{aligned}
$$


From Lieberman [15, Theorem 2], we can find $\theta \in(0,1)$ and $M_{1}>0$ such that

$$
\widehat{u}_{n} \in C^{1, \theta}(\bar{\Omega}) \quad \text { and } \quad\left\|\widehat{u}_{n}\right\|_{C^{1, \theta}(\bar{\Omega})} \leq M_{1}, \quad \text { for all } n \geq 1
$$

From the compact embedding of $C^{1, \theta}(\bar{\Omega})$ into $C^{1}(\bar{\Omega})$ and (3.13), we see that we may assume that

$$
\widehat{u}_{n} \rightarrow u_{*} \quad \text { in } C^{1}(\bar{\Omega}) .
$$

By contradiction, we suppose that $u_{*}=0$. Then, we can find $n_{0} \geq 1$ such that $\widehat{u}_{n}(z) \in\left(0, \delta_{0}\right]$ for all $z \in \Omega$ and all $n \geq n_{0}$. In particular, by $\left(\mathrm{H}_{1}\right)(\mathrm{d})$, we have

$$
f(z, x) \geq 0, \quad \text { for a.a. } z \in \Omega, \text { all } x \in\left[0, \widehat{u}_{n}(z)\right] \text { and all } n \geq n_{0} .
$$

Since $\widehat{u}_{n} \in \operatorname{int} C_{+}$, we can find $t_{n}>0$ such that $t_{n} \widehat{u}_{\lambda} \leq \widehat{u}_{n}$, where $\widehat{u}_{\lambda}$ is the unique positive solution of problem (3.9) (see, Marano and Papageorgiou in [17]). Let $t_{n}>0$ be the biggest such positive numbers and suppose that $t_{n} \in(0,1)$. For $\delta>0$, let $\widehat{u}_{\lambda}^{\delta}=\widehat{u}_{\lambda}+\delta \in \operatorname{int} C_{+}$. Let $\rho=\|\bar{u}\|_{\infty}$ and let $\xi_{\rho}>0$ be as postulated by hypothesis $\left(\mathrm{H}_{1}\right)(\mathrm{e})$. Bearing in mind (3.9) and Proposition 3.3 as well as that $q<p$, we can find $\mu(\delta) \rightarrow 0^{+}$as $\delta \rightarrow 0^{+}$, and $\delta^{*}>0$ such that, for all $n \geq n_{0}$, we have

$$
\begin{array}{rlr}
-\Delta_{p}\left(t_{n} \widehat{u}_{\lambda}^{\delta}\right) & +\left(\beta(z)+\xi_{\rho}\right)\left(t_{n} \widehat{u}_{\lambda}^{\delta}\right)^{p-1} \\
\leq & t_{n}^{p-1}\left[-\Delta_{p}\left(\widehat{u}_{\lambda}\right)+\left(\beta(z)+\xi_{\rho}\right) \widehat{u}_{\lambda}^{p-1}\right] & \\
= & t_{n}^{p-1} \lambda \widehat{u}_{\lambda}^{q-1}+\xi_{\rho}\left(t_{n} \widehat{u}_{\lambda}\right)^{p-1}+\mu(\delta) & \\
= & \lambda\left(t_{n} \widehat{u}_{\lambda}\right)^{q-1}+\xi_{\rho}\left(t_{n} \widehat{u}_{\lambda}\right)^{p-1}+\lambda\left(t_{n}^{p-1}-t_{n}^{q-1}\right) \widehat{u}_{\lambda}^{q-1}+\mu(\delta) & \\
\leq & \lambda\left(t_{n} \widehat{u}_{\lambda}\right)^{q-1}+\xi_{\rho}\left(t_{n} \widehat{u}_{\lambda}\right)^{p-1} & \\
\leq & \lambda\left(t_{n} \widehat{u}_{\lambda}\right)^{q-1}+f\left(z, t_{n} \widehat{u}_{\lambda}\right)+\xi_{\rho}\left(t_{n} \widehat{u}_{\lambda}\right)^{p-1} & \\
\leq & \lambda \widehat{u}_{n}^{q-1}+f\left(z, \widehat{u}_{n}\right)+\xi_{\rho} \widehat{u}_{n}^{p-1} & \text { a.e. in } \Omega . \\
& =-\Delta_{p} \widehat{u}_{n}+\left(\beta(z)+\xi_{\rho}\right) \widehat{u}_{n}^{p-1}(z),
\end{array}
$$

From this, by standard arguments, we infer that $t_{n} \widehat{u}_{\lambda}^{\delta} \leq \widehat{u}_{n}$ for all $n \geq n_{0}$ and all $\delta \in\left(0, \delta^{*}\right]$ which implies that $\widehat{u}_{n}-t_{n} \widehat{u}_{\lambda} \in \operatorname{int} C_{+}$and this contradicts the maximality of $t_{n}>0$. Therefore, we have $t_{n} \geq 1$ for all $n \geq 1$. Hence, one has $\widehat{u}_{\lambda} \leq \widehat{u}_{n}$ for all $n \geq 1$. So, it results that $0<\widehat{u}_{\lambda} \leq u_{*}$, i.e. $u_{*}>0$, again a contradiction. Therefore, $u_{*} \neq 0$ and $u_{*} \in \operatorname{int} C_{+}$and $\widehat{u}_{\lambda} \leq u_{*}$. Moreover, since

$$
A\left(\widehat{u}_{n}\right)+\beta \widehat{u}_{n}^{p-1}=\lambda \widehat{u}_{n}^{q-1}+N_{f}\left(\widehat{u}_{n}\right), \quad \text { for all } n \geq 1,
$$

passing to the limit as $n \rightarrow \infty$ and using (3.14), we obtain

$$
A\left(u_{*}\right)+\beta u_{*}^{p-1}=\lambda u_{*}^{q-1}+N_{f}\left(u_{*}\right),
$$


so $u_{*} \in \operatorname{int} C_{+}$is a solution of $\left(\mathrm{P}_{\lambda}\right)$. Clearly, $u_{*} \in \operatorname{int} C_{+}$is the smallest nontrivial positive solution of $\left(\mathrm{P}_{\lambda}\right)$. Similarly, we produce $v_{*} \in-\operatorname{int} C_{+}$the biggest nontrivial negative solution of $\left(\mathrm{P}_{\lambda}\right)$.

Proposition 3.6. If hypotheses $\mathrm{H}(\beta)$ and $\left(\mathrm{H}_{1}\right)$ hold and $\lambda \in\left(0, \lambda^{*}\right)$, then problem $\left(\mathrm{P}_{\lambda}\right)$ has two more nontrivial constant sign solutions:

$$
\widehat{u} \in \operatorname{int} C_{+}, \quad \widehat{v} \in-\operatorname{int} C_{+} \quad \text { and } \quad u_{0} \leq \widehat{u}, \quad u_{0} \neq \widehat{u}, \quad \widehat{v} \leq v_{0}, \quad \widehat{v} \neq v_{0} .
$$

Proof. From Proposition 3.2 we know that problem $\left(\mathrm{P}_{\lambda}\right)$ has a nontrivial solution $u_{0}$ in the order interval

$$
[0, \bar{u}]=\left\{u \in W^{1, p}(\Omega): 0 \leq u(z) \leq \bar{u}(z) \text { a.e. in } \Omega\right\} .
$$

We may assume that $u_{0}$ is the only nontrivial solution of $\left(\mathrm{P}_{\lambda}\right)$ in $[0, \bar{u}]$ (otherwise we already have a second nontrivial solution of $\left(\mathrm{P}_{\lambda}\right)$ and the two solutions are ordered, since the solution set is downward directed). Using $u_{0} \in \operatorname{int} C_{+}$, we introduce the following truncation of the reaction in problem $\left(\mathrm{P}_{\lambda}\right)$ :

$$
\left(l_{\lambda}\right)_{u_{0}}(z, x)= \begin{cases}\lambda u_{0}^{q-1}(z)+f\left(z, u_{0}(z)\right) & \text { if } x<u_{0}(z), \\ \lambda x^{q-1}+f(z, x) & \text { if } x \geq u_{0}(z) .\end{cases}
$$

This is a Carathéodory function and, using the notation adopted is Section 2, we set

$$
\left(\Phi_{\lambda}\right)_{u_{0}}(u)=\frac{\sigma(u)}{p}-\int_{\Omega}\left(L_{\lambda}\right)_{u_{0}}(z, u(z)) d z, \quad \text { for all } u \in W^{1, p}(\Omega) .
$$

Claim 1. The function $\left(\Phi_{\lambda}\right)_{u_{0}}$ satisfies the C-condition.

Let $\left\{u_{n}\right\} \in W^{1, p}(\Omega)$ be a sequence such that

$$
\left|\left(\Phi_{\lambda}\right)_{u_{0}}\left(u_{n}\right)\right| \leq M_{2}, \quad \text { for some } M_{2}>0, \text { for all } n \geq 1,
$$

and

$$
\left(1+\left\|u_{n}\right\|\right)\left(\left(\Phi_{\lambda}\right)_{u_{0}}^{\prime}\left(u_{n}\right)\right) \rightarrow 0^{+} \quad \text { in } W^{-1, p^{\prime}}(\Omega), \text { as } n \rightarrow \infty .
$$

From the latter, we have

$$
\left|\left\langle\left(\Phi_{\lambda}\right)_{u_{0}}^{\prime}\left(u_{n}\right), v\right\rangle\right| \leq \frac{\varepsilon_{n}}{1+\left\|u_{n}\right\|}\|v\|, \quad \text { for all } v \in W^{1, p}(\Omega), \text { with } \varepsilon_{n} \downarrow 0^{+},
$$

that is,

$$
\left.\left|\left\langle A\left(u_{n}\right), v\right\rangle+\int_{\Omega} \beta\right| u_{n}\right|^{p-2} u_{n} v d z-\int_{\Omega}\left(l_{\lambda}\right)_{u_{0}}\left(z, u_{n}\right) v d z \mid \leq \frac{\varepsilon_{n}}{1+\left\|u_{n}\right\|}\|v\| .
$$

Putting $v=-u_{n}^{-} \in W^{1, p}(\Omega)$, from the previous inequality, an easy computation gives $\sigma\left(u_{n}^{-}\right) \leq \varepsilon_{n}$ for all $n \geq 1$, which, by Lemma 2.4, clearly ensures that

$$
u_{n}^{-} \rightarrow 0 \quad \text { in } W^{1, p}(\Omega) \text {. }
$$


Next, in (3.19), we choose $v=u_{n}^{+} \in W^{1, p}(\Omega)$. Then, because $u_{0} \in \operatorname{int} C_{+}$, see also (3.15), we have

$$
-\sigma\left(u_{n}^{+}\right)+\lambda \int_{\left\{u_{n}>u_{0}\right\}} u_{n}^{q}+\int_{\left\{u_{n}>u_{0}\right\}} f\left(z, u_{n}^{+}\right) u_{n}^{+} d z \leq M_{3},
$$

for some $M_{3}>0$. On the other hand, from (3.17) and (3.20) we get

$$
\sigma\left(u_{n}^{+}\right)-\lambda \frac{p}{q} \int_{\left\{u_{n}>u_{0}\right\}} u_{n}{ }^{q}-\int_{\left\{u_{n}>u_{0}\right\}} p F\left(z, u_{n}\right) d z \leq M_{4}
$$

for some $M_{4}>0$ and all $n \geq 1$. Adding (3.21) and 3.22, we obtain

$$
\int_{\Omega}\left[f\left(z, u_{n}^{+}\right) u_{n}^{+}-p F\left(z, u_{n}^{+}\right) d z\right] d z \leq M_{5}+\lambda\left(\frac{p}{q}-1\right) \int_{\Omega}\left(u_{n}^{+}\right)^{q} d z
$$

for some $M_{5}>0$ and all $n \geq 1$. From hypothesis $\left(\mathrm{H}_{1}\right)(\mathrm{c})$, there exist $\xi_{1} \in\left(0, \xi_{0}\right)$ and $M_{6}>0$ such that

$$
\xi_{1}|x|^{\tau} \leq f(z, x) x-p F(z, x), \quad \text { for a.a. } z \in \Omega \text { and all }|x| \geq M_{6} .
$$

On the other hand, hypothesis $\left(\mathrm{H}_{1}\right)(\mathrm{a})$ implies that there exists $M_{7}>0$ such that

$$
|f(z, x) x-p F(z, x)| \leq M_{4}, \quad \text { for a.a. } z \in \Omega \text { and all }|x| \leq M_{6} .
$$

Combining (3.24) and (3.25), one has

$$
\xi_{1}|x|^{\tau}-M_{8} \leq f(z, x) x-p F(z, x), \quad \text { for a.a. } z \in \Omega \text { and all } x \geq 0 .
$$

Returning to (3.23) and using (3.26), we obtain

$$
\int_{\Omega}\left(u_{n}^{+}\right)^{\tau} d z \leq M_{9}+\lambda\left(\frac{p}{q}-1\right) \int_{\Omega}\left(u_{n}^{+}\right)^{q} d z
$$

for some $M_{9}>0$ and all $n \geq 1$. Since $q<\tau \leq p$, from (3.27) we infer that

$$
\left\{u_{n}^{+}\right\} \subseteq L^{\tau}(\Omega) \text { is bounded. }
$$

First suppose that $N \neq p$. Recall that $\tau \leq p<p^{*}$. So, we can find $t \in[0,1)$ such that

$$
\frac{1}{p}=\frac{1-t}{\tau}+\frac{t}{p^{*}}
$$

Invoking the interpolation inequality (see, for example, Gasinski-Papageorgiou [7, p. 905]) we have

$$
\left\|u_{n}^{+}\right\|_{p} \leq\left\|u_{n}^{+}\right\|_{\tau}^{1-t}\left\|u_{n}^{+}\right\|_{p^{*}}^{t}, \quad \text { for all } n \geq 1,
$$

which implies that

$$
\left\|u_{n}^{+}\right\|_{p}^{p} \leq M_{10}\left\|u_{n}^{+}\right\|^{t p}, \quad \text { for some } M_{10}>0 \text { and for all } n \geq 1 .
$$

Moreover, from (3.19) with $v=u_{n}^{+} \in W^{1, p}(\Omega)$, we have

$$
\left|\sigma\left(u_{n}^{+}\right)-\int_{\Omega}\left(l_{\lambda}\right)_{u_{0}}\left(z, u_{n}\right) u_{n}^{+}\right| \leq \varepsilon_{n}, \quad \text { for all } n \geq 1 .
$$


But from $\left(\mathrm{H}_{1}\right)(\mathrm{a})$, (b) and (3.16) we have

$$
\left|x\left(l_{\lambda}\right)_{u_{0}}(z, x)\right| \leq c_{4}\left(1+|x|^{p}\right), \quad \text { for a.a. } z \in \Omega \text {, all } x \in \mathbb{R} \text {, some } c_{4}>0 .
$$

Therefore, owing to Lemma 2.4, exploiting (3.30), (3.31) and (3.29), we can assert that

$$
c_{0}\left\|u_{n}^{+}\right\|^{p} \leq c_{5}\left(1+\left\|u_{n}^{+}\right\|_{p}^{p}\right) \leq c_{6}\left(1+\left\|u_{n}^{+}\right\|^{t p}\right),
$$

for some $c_{5}, c_{6}>0$ and all $n \geq 1$. Recall that $t \in[0,1)$. So $t p<p$ and from the previous inequality, it follows that

$$
\left\{u_{n}^{+}\right\} \subseteq W^{1, p}(\Omega) \text { is bounded. }
$$

Now, suppose that $N=p$. In this case $p^{*}=+\infty$ and by the Sobolev embedding theorem one has that $W^{1, p}(\Omega) \hookrightarrow L^{r}(\Omega)$ for all $r \in[1,+\infty)$. So, in the above argument we replace $p^{*}$ with $r \in[1,+\infty)$ big and again we reach (3.33).

Putting together (3.20) and (3.33) we infer that $\left\{u_{n}\right\} \subset W^{1, p}(\Omega)$ is bounded. Consequently, we may assume that

$$
u_{n} \rightarrow u \quad \text { in } W^{1, p}(\Omega) \quad \text { and } \quad u_{n} \rightarrow u \quad \text { in } L^{p}(\Omega) .
$$

In (3.19), we let $v=u_{n}-u \in W^{1, p}(\Omega)$, pass to the limit as $n \rightarrow+\infty$ and use (3.34), we can realize that

$$
\lim _{n \rightarrow+\infty}\left\langle A\left(u_{n}\right), u_{n}-u\right\rangle=0,
$$

and since $A$ is an operator of type $S_{+}$, see Proposition 2.3, we obtain that $u_{n} \rightarrow u$ in $W^{1, p}(\Omega)$ and this proves Claim 1.

Claim $2 u_{0} \in \operatorname{int} C_{+}$is a local minimizer of $\left(\Phi_{\lambda}\right)_{u_{0}}$.

To this end we use the following truncation of $l_{\lambda}(z, x)$ :

$$
\left(l_{\lambda}\right)_{u_{0}}^{\bar{u}}(z, x):= \begin{cases}l_{\lambda}\left(z, u_{0}(z)\right) & \text { if } x<\underline{u}_{0}(z), \\ l_{\lambda}(z, x) & \text { if } u_{0}(z) \leq x \leq \bar{u}(z), \\ l_{\lambda}(z, \bar{u}(z)) & \text { if } x>\bar{u}(z) .\end{cases}
$$

According to Lemmas 2.5 and 2.6, we point out that the functional

$$
\left(\Phi_{\lambda}\right)_{u_{0}}^{\bar{u}}: W^{1, p}(\Omega) \rightarrow \mathbb{R}
$$

defined by

$$
\left(\Phi_{\lambda}\right)_{u_{0}}^{\bar{u}}(u)=\frac{\sigma(u)}{p}-\int_{\Omega}\left(L_{\lambda}\right)_{u_{0}}^{\bar{u}}(z, u(z)) d z, \quad \text { for all } u \in W^{1, p}(\Omega),
$$

admits a global minimum $\widehat{u}_{0} \in W^{1, p}(\Omega)$ such that $u_{0} \leq \widehat{u}_{0} \leq \bar{u}$. But recall that we have assumed that $u_{0}$ is the only nontrivial solution of $\left(\mathrm{P}_{\lambda}\right)$ in the order interval $[0, \bar{u}]$, it follows that $u_{0} \leq \widehat{u}_{0}=u_{0}$.

From the proof of Proposition 3.2, we have that $\bar{u}-u_{0} \in \operatorname{int} C_{+}$. Hence, note that $\left(\Phi_{\lambda}\right)_{u_{0}}^{\bar{u}}(u)=\left(\Phi_{\lambda}\right)_{u_{0}}(u)$ for all $u \in[0, \bar{u}]$, we deduce that $u_{0}$ is a $C^{1}(\bar{\Omega})$ 
local minimizer of $\left(\Phi_{\lambda}\right)_{u_{0}}$ and, owing to Proposition 2.2, $W^{1, p}(\Omega)$-minimizer of $\left(\Phi_{\lambda}\right)_{u_{0}}$. So, Claim 2 is verified.

It is not restrictive to assume that $u_{0}$ is an isolated element of $K_{\left(\Phi_{\lambda}\right)_{u_{0}}}$. Otherwise, we obtain a sequence of distinct nontrivial positive solutions of $\left(\mathrm{P}_{\lambda}\right)$ belonging to int $C_{+}$, see Lemma 2.5. So, we assume that $u_{0}$ is an isolated critical point of the functional $\left(\Phi_{\lambda}\right)_{u_{0}}$ which is a local minimizer (see Claim 2). Therefore, we can find $\rho \in(0,1)$ small such that

$$
\left(\Phi_{\lambda}\right)_{u_{0}}\left(u_{0}\right)<\inf \left[\left(\Phi_{\lambda}\right)_{u_{0}}(u):\left\|u-u_{0}\right\|=\rho\right]=m_{\lambda}^{+} .
$$

For $t>0$, we have

$$
\begin{aligned}
\left(\Phi_{\lambda}\right)_{u_{0}}\left(t \widehat{u}_{1}\right) & =\frac{t^{p}}{p} \sigma\left(\widehat{u}_{1}\right)-\int_{\Omega}\left(L_{\lambda}\right)_{u_{0}}\left(z, t \widehat{u}_{1}(z)\right) d z \\
& =\frac{t^{p}}{p} \widehat{\lambda}_{1}(\beta)-\int_{\Omega}\left(L_{\lambda}\right)_{u_{0}}\left(z, t \widehat{u}_{1}(z)\right) d z \\
& \leq \frac{t^{p}}{p} \widehat{\lambda}_{1}(\beta)-\frac{\lambda}{q} t^{q}\left\|\widehat{u}_{1}\right\|_{q}^{q}-\int_{\Omega} F\left(z, t \widehat{u}_{1}(z)\right) d z+M_{11},
\end{aligned}
$$

for some $M_{11}>0$. Hypothesis $\left(\mathrm{H}_{1}\right)(\mathrm{b})$ implies that, given $\varepsilon>0$, we can find $M_{12}=M_{12}(\varepsilon)$ such that

$$
F(z, x) \geq \frac{1}{p}(\eta(z)-\varepsilon) x^{p}, \quad \text { for a.a. } z \in \Omega \text { and } x \geq M_{12} .
$$

Using the above estimates, for some $M_{13}>0$, we have

$$
\begin{aligned}
\left(\Phi_{\lambda}\right)_{u_{0}}\left(t \widehat{u}_{1}\right) & \leq \frac{t^{p}}{p} \widehat{\lambda}_{1}(\beta)-\frac{\lambda t^{q}}{q}\left\|\widehat{u}_{1}\right\|_{q}^{q}-\frac{t^{p}}{p} \int_{\Omega} \eta \widehat{u}_{1}^{p} d z+\frac{\varepsilon t^{p}}{p}+M_{13} \\
& \leq \frac{t^{p}}{p}\left[\int_{\Omega}\left(\widehat{\lambda}_{1}(\beta)-\eta(z) \widehat{u}_{1}(z)^{p}\right) d z+\varepsilon\right]+M_{13} .
\end{aligned}
$$

Since $\widehat{u}_{1} \in \operatorname{int} C_{+}$and $\eta(z) \geq \widehat{\lambda}_{1}(\beta)$ almost everywhere in $\Omega, \eta \neq \widehat{\lambda}_{1}(\beta)$, we have

$$
\varepsilon_{*}=\int_{\Omega}\left(\eta(z)-\widehat{\lambda}_{1}(\beta)\right) \widehat{u}_{1}^{p}(z) d z>0
$$

and

$$
\left(\Phi_{\lambda}\right)_{u_{0}}\left(t \widehat{u}_{1}\right) \leq \frac{t^{p}}{p}\left[-\varepsilon_{*}+\varepsilon\right]+M_{13}
$$

Choosing $\varepsilon \in\left(0, \varepsilon_{*}\right)$, from the above inequality, we infer that

$$
\left(\Phi_{\lambda}\right)_{u_{0}}\left(t \widehat{u}_{1}\right) \rightarrow-\infty \text { as } t \rightarrow+\infty .
$$

Claim 1, (3.36) and (3.37) permit the use of Theorem 2.1 (the mountain pass theorem) and we obtain for the functional $\left(\Phi_{\lambda}\right)_{u_{0}}$ a critical point $\widehat{u} \in W^{1, p}(\Omega)$ such that

$$
\left(\Phi_{\lambda}\right)_{u_{0}}\left(u_{0}\right)<m_{\lambda}^{+} \leq\left(\Phi_{\lambda}\right)_{u_{0}}(\widehat{u}) .
$$

Clearly $\widehat{u} \neq u_{0}$ and, by Lemma 2.6, we have $u_{0} \leq \widehat{u}$ and so $\widehat{u}$ is a second nontrivial positive solution of $\left(\mathrm{P}_{\lambda}\right)$. Also we have that $\widehat{u} \in \operatorname{int} C_{+}$. 
Similarly, starting with $v_{0} \in-\operatorname{int} C_{+}$and working as above we produce a second nontrivial negative solution $\widehat{v} \in-\operatorname{int} C_{+}$of $\left(\mathrm{P}_{\lambda}\right)$ such that $\widehat{v} \leq v_{0}$ with $\widehat{v} \neq v_{0}$.

\section{Nodal solutions}

In this section, we turn out our attention to the existence of nodal (i.e. sign changing) solutions of $\left(\mathrm{P}_{\lambda}\right)$. We start by computing the critical groups of $\Phi_{\lambda}$ at the origin.

Proposition 4.1. If hypotheses $\mathrm{H}(\beta)$ and $\left(\mathrm{H}_{1}\right)$ hold and $\lambda>0$, then

$$
C_{k}\left(\Phi_{\lambda}, 0\right)=0, \quad \text { for all } k \geq 0 \text {. }
$$

Proof. By virtue of hypothesis $\left(\mathrm{H}_{1}\right)(\mathrm{d})$ for $\eta \in(q, p)$, for almost all $z \in \Omega$, all $|x| \leq \delta_{0}$, we have

$$
\begin{aligned}
\eta\left(\frac{\lambda}{q}|x|^{q}+F(z, x)\right)- & \left(\lambda|x|^{q}+f(z, x) x\right)=\lambda\left(\frac{\eta}{q}-1\right)|x|^{q}+\eta F(z, x)-f(z, x) x \\
& \geq \lambda\left(\frac{\eta}{q}-1\right)|x|^{q}-f(z, x) x \geq \lambda\left(\frac{\eta}{q}-1\right)|x|^{q}-c_{1}|x|^{r} .
\end{aligned}
$$

Since $\eta>q$ and $q<r$, we can use Proposition 2.1 of Jiu-Su ([13]) and conclude that $C_{k}\left(\Phi_{\lambda}, 0\right)=0$ for all $k \geq 0$.

Let $u_{*} \in \operatorname{int} C_{+}$and $v_{*} \in-\operatorname{int} C_{+}$be the two extremal nontrivial constant solutions, we introduce the following truncation of the reaction in $\left(\mathrm{P}_{\lambda}\right)$ :

$$
\left(l_{\lambda}\right)_{v_{*}}^{u_{*}}(z, x)= \begin{cases}\lambda\left|v_{*}(z)\right|^{q-2} v_{*}(z)+f\left(z, v_{*}(z)\right) & \text { if } x<v_{*}(z), \\ \lambda|x|^{q-2} x+f(z, x) & \text { if } v_{*}(z) \leq x \leq u_{*}(z), \\ \lambda\left|u_{*}(z)\right|^{q-2} u_{*}(z)+f\left(z, u_{*}(z)\right) & \text { if } u_{*}(z)<x\end{cases}
$$

we set

$$
\left(L_{\lambda}\right)_{v^{*}}^{u^{*}}(z, x)=\int_{0}^{x}\left(l_{\lambda}\right)_{v_{*}}^{u_{*}}(z, s) d s
$$

and consider the $C^{1}$-functional $\left(\Phi_{\lambda}\right)_{v^{*}}^{u^{*}}: W^{1, p}(\Omega) \rightarrow \mathbb{R}$ defined by

$$
\left(\Phi_{\lambda}\right)_{v^{*}}^{u^{*}}(u)=\frac{1}{p} \sigma(u)-\int_{\Omega}\left(L_{\lambda}\right)_{v^{*}}^{u^{*}}(z, u(z)) d z, \quad \text { for all } u \in W^{1, p}(\Omega) .
$$

Proposition 4.2. If hypotheses $\mathrm{H}(\beta)$ and $\left(\mathrm{H}_{1}\right)$ hold and $\lambda>0$, then

$$
C_{k}\left(\left(\Phi_{\lambda}\right)_{v^{*}}^{u^{*}}, 0\right)=0, \quad \text { for all } k \geq 0 \text {. }
$$

Proof. We consider the homotopy

$$
h_{\lambda}(t, u)=(1-t)\left(\Phi_{\lambda}\right)_{v^{*}}^{u^{*}}(u)+t \Phi_{\lambda}(u), \quad \text { for all }(t, u) \in[0,1] \times W^{1, p}(\Omega) .
$$

Evidently, $\left(\Phi_{\lambda}\right)_{v^{*}}^{u^{*}}$ is coercive and standard arguments prove that it satisfies the C-condition. Moreover, a simple modification of the proof of Claim 1 in the proof of Proposition 3.6 shows that the functional $\Phi_{\lambda}$ satisfies the C-condition. 
Claim. We may assume that there exists $\rho \in(0,1)$ small enough such that $u=0$ is the only critical point of $h_{\lambda}(t, \cdot)_{t \in[0,1]}$ in $\bar{B}_{\rho}=\left\{u \in W^{1, p}(\Omega):\|u\| \leq \rho\right\}$. Indeed, suppose we can find $\left\{t_{n}\right\}_{n \geq 1} \subseteq[0,1]$ and $\left\{u_{n}\right\}_{n \geq 1} \subseteq W^{1, p}(\Omega)$ such that (4.2) $t_{n} \rightarrow t, \quad u_{n} \rightarrow 0 \quad$ in $W^{1, p}(\Omega) \quad$ and $\quad\left(h_{\lambda}\right)_{u}^{\prime}\left(t_{n}, u_{n}\right)=0, \quad$ for all $n \geq 1$.

From the equation in (4.2), we have

$$
A\left(u_{n}\right)+\beta\left|u_{n}\right|^{p-2} u_{n}=\left(1-t_{n}\right) N_{\left(l_{\lambda}\right)_{v *}^{u_{*}}}\left(u_{n}\right)+t_{n}\left(\lambda\left|u_{n}\right|^{q-2} u_{n}+N_{f}\left(u_{n}\right)\right)
$$

for all $n \geq 1$, hence

$$
\begin{array}{cc}
-\Delta_{p} u_{n}(z)+\beta(z)\left|u_{n}(z)\right|^{p-2} u_{n}(z)=\left(1-t_{n}\right)\left(l_{\lambda}\right) v_{v_{*}}^{u_{*}}(z, x) & \\
+\lambda t_{n}\left|u_{n}(z)\right|^{q-2} u_{n}(z)+t_{n} f\left(z, u_{n}(z)\right) & \text { a.e. in } \Omega, \\
\frac{\partial u_{n}}{\partial n}=0 & \text { on } \partial \Omega .
\end{array}
$$

From $\mathrm{Hu}$ and Papageorgiou [11], we know that we can find $\theta \in(0,1)$ and $M_{14}>0$ such that

$$
u_{n} \in C^{1, \theta}(\bar{\Omega}) \quad \text { and } \quad\left\|u_{n}\right\|_{C^{1, \theta}(\bar{\Omega})} \leq M_{14}, \quad \text { for all } n \geq 1 .
$$

Since $C^{1, \theta}(\bar{\Omega})$ is embedded compactly in $C^{1}(\bar{\Omega})$ and by virtues of (4.2) and (4.4), we have $u_{n} \rightarrow 0$ in $C^{1}(\bar{\Omega})$, hence

$u_{n} \in\left[v_{*}, u_{*}\right]=\left\{u \in W^{1, p}(\Omega): v_{*}(z) \leq u(z) \leq u_{*}(z)\right.$ a.e. in $\left.\Omega\right\} \quad$ for all $n \geq n_{0}$.

Thus, for $n \geq n_{0}$, equation (4.3) becomes

$$
\begin{aligned}
-\Delta_{p} u_{n}(z)+\beta(z)\left|u_{n}(z)\right|^{p-2} u_{n}(z) & =\lambda\left|u_{n}(z)\right|^{q-2} u_{n}(z)+f\left(z, u_{n}(z)\right) \text { a.e. in } \Omega, \\
\frac{\partial u_{n}}{\partial n} & =0 \quad \text { on } \partial \Omega,
\end{aligned}
$$

then $\left\{u_{n}\right\}_{n \geq 1} \subseteq\left[v_{*}, u_{*}\right] \cap C^{1}(\bar{\Omega})$ are nontrivial nodal solutions of $\left(\mathrm{P}_{\lambda}\right)$. So, we are done. This proves the claim.

The above claim, the homotopy invariance of the critical groups and Proposition 4.1 , for all $k \geq 0$, imply that

$$
\begin{aligned}
C_{k}\left(h_{\lambda}(0, \cdot), 0\right)=C_{k}\left(h_{\lambda}(1, \cdot), 0\right) & \Rightarrow C_{k}\left(\left(\Phi_{\lambda}\right)_{v^{*}}^{u^{*}}, 0\right)=C_{k}\left(\Phi_{\lambda}, 0\right) \\
& \Rightarrow C_{k}\left(\left(\Phi_{\lambda}\right)_{v^{*}}^{u^{*}}, 0\right)=0 .
\end{aligned}
$$

Now, we are ready for the complete multiplicity result theorem for problem $\left(\mathrm{P}_{\lambda}\right)$. We stress that our result provides sign information for all the solutions produced.

THEOREM 4.3. If hypotheses $\mathrm{H}(\beta)$ and $\left(\mathrm{H}_{1}\right)$ hold, then there exists $\lambda^{*}>0$ such that for all $\lambda \in\left(0, \lambda^{*}\right)$ problem $\left(\mathrm{P}_{\lambda}\right)$ has at least five solutions:

$\widehat{u} \in \operatorname{int} C_{+}, \quad \widehat{v} \in-\operatorname{int} C_{+}, \quad u_{0} \leq \widehat{u}, \quad u_{0} \neq \widehat{u}, \quad \widehat{v} \leq v_{0}, \quad \widehat{v} \neq v_{0}$, and $\left.y_{0} \in\right] v_{0}, u_{0}\left[\cap C^{1}(\bar{\Omega})\right.$ nodal. 
Proof. From Proposition 3.6, we know that there exists $\lambda^{*}>0$ such that for all $\lambda \in\left(0, \lambda^{*}\right)$ problem $\left(\mathrm{P}_{\lambda}\right)$ has at least four nontrivial solutions of constant $\operatorname{sign}$

$$
\begin{array}{ll}
u_{0}, \widehat{u} \in \operatorname{int} C_{+}, & u_{0} \leq \widehat{u}, \quad u_{0} \neq \widehat{u}, \\
v_{0}, \widehat{v} \in-\operatorname{int} C_{+}, & \widehat{v} \leq v_{0}, \quad v_{0} \neq \widehat{v} .
\end{array}
$$

We may assume that the solutions $u_{0}$ and $v_{0}$ are extremal, i.e. $u_{0}=u_{*}$ and $v_{0}=v_{*}$ (see Proposition 3.5)

We consider the following truncations of the reaction term of $\left(\mathrm{P}_{\lambda}\right)$ :

$$
\begin{aligned}
& \left(l_{\lambda}\right)_{0}^{u_{*}}(z, x)= \begin{cases}0 & \text { if } x<0, \\
\lambda x^{q-1}+f(z, x) & \text { if } 0 \leq x \leq u_{*}(z), \\
\lambda u_{*}^{q-1}(z)+f\left(z, u_{*}(z)\right) & \text { if } x>u_{*}(z),\end{cases} \\
& \left(l_{\lambda}\right)_{v_{*}}^{0}(z, x)= \begin{cases}\lambda\left|v_{*}(z)\right|^{q-2} v_{*}(z)+f\left(z, v_{*}(z)\right) & \text { if } x<v_{*}(z), \\
\lambda|x|^{q-2} x+f(z, x) & \text { if } v_{*}(z) \leq x \leq 0, \\
0 & \text { if } x>0,\end{cases} \\
& \left(l_{\lambda}\right)_{v_{*}}^{u_{*}}(z, x)= \begin{cases}\lambda\left|v_{*}(z)\right|^{q-2} v_{*}(z)+f\left(z, v_{*}(z)\right) & \text { if } x<v_{*}(z), \\
\lambda|x|^{q-2} x+f(z, x) & \text { if } v_{*}(z) \leq x \leq u_{*}(z), \\
\lambda u_{*}^{q-1}(z)+f\left(z, u_{*}(z)\right) & \text { if } x>u_{*}(z),\end{cases}
\end{aligned}
$$

and the correspondent associate functionals, see the notation adopted in Section 2 ,

$$
\begin{aligned}
& \left(\Phi_{\lambda}\right)_{0}^{u_{*}}(u)=\frac{\sigma(u)}{p}-\int_{\Omega}\left(L_{\lambda}\right)_{0}^{u_{*}}(z, u(z)) d z, \\
& \left(\Phi_{\lambda}\right)_{v_{*}}^{0}(u)=\frac{\sigma(u)}{p}-\int_{\Omega}\left(L_{\lambda}\right)_{v_{*}}^{0}(z, u(z)) d z \\
& \left(\Phi_{\lambda}\right)_{v_{*}}^{u_{*}}(u)=\frac{\sigma(u)}{p}-\int_{\Omega}\left(L_{\lambda}\right)_{v_{*}}^{u_{*}}(z, u(z)) d z
\end{aligned}
$$

Claim 1. $K_{\left(\Phi_{\lambda}\right)_{0}^{u_{*}}}=\left\{0, u_{*}\right\}$ and $K_{\left(\Phi_{\lambda}\right)_{v_{*}}^{0}}=\left\{v_{*}, 0\right\}$.

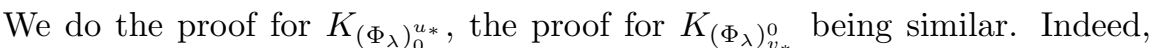
bearing in mind Lemmata 2.5 and 2.6, it is easy to see that $K_{\left(\Phi_{\lambda}\right)_{0}^{u_{*}} \subseteq}^{\subseteq}\left[0, u_{*}\right]$. The extremality of $u_{*}$ implies that $K_{\left(\Phi_{\lambda}\right)_{0}^{u *}}=\left\{0, u_{*}\right\}$. Similarly, we show that

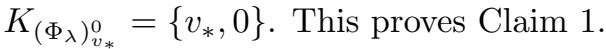

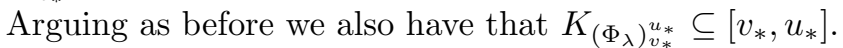

ClaIm 2. $u_{*} \in \operatorname{int} C_{+}$and $v_{*} \in-\operatorname{int} C_{+}$are local minimizer of $\left(\Phi_{\lambda}\right)_{v_{*}}^{u_{*}}$.

Using hypothesis $\mathrm{H}_{1}(\mathrm{~d})$ and since $q<p$ and $\beta \in L^{\infty}(\Omega)$, by $\left(\mathrm{l}_{1}\right)$ of Lemma 2.5, we have that there exists $\widehat{u} \in W^{1, p}(\Omega)$ with $\widehat{u} \neq 0$ such that

$$
\left(\Phi_{\lambda}\right)_{0}^{u_{*}}(\widehat{u})=\inf \left\{\left(\Phi_{\lambda}\right)_{0}^{u_{*}}(u): u \in W^{1, p}(\Omega)\right\} .
$$


Also $\widehat{u} \in\left\{0, u_{*}\right\}$, see Claim 1. Hence, $\widehat{u}=u_{*} \in \operatorname{int} C_{+}$. Therefore, there exists a neighbourhood $U$ in $\operatorname{int} C^{+}$of $u_{*}$ such that

$$
\left(\Phi_{\lambda}\right)_{v_{*}}^{u_{*}}\left(u_{*}\right)=\left(\Phi_{\lambda}\right)_{0}^{u_{*}}\left(u_{*}\right) \leq\left(\Phi_{\lambda}\right)_{0}^{u_{*}}(u)=\left(\Phi_{\lambda}\right)_{v_{*}}^{u_{*}}(u), \quad \text { for all } u \in U
$$

This means that $u_{*}$ is a local $C^{1}(\Omega)$-minimizer of $\left(\Phi_{\lambda}\right)_{v_{*}}^{u_{*}}$, so, by Proposition 2.2, $u_{*}$ is a local $W^{1, p}(\Omega)$-minimizer of $\left(\Phi_{\lambda}\right)_{v_{*}}^{u_{*}}$. Similarly, for $v_{*} \in-\operatorname{int} C_{+}$, using this time the functional $\left(\Phi_{\lambda}\right)_{v_{*}}^{0}$. This proves Claim 2 .

Without any loss of generality, we may assume that

$$
\left(\Phi_{\lambda}\right)_{v_{*}}^{u_{*}}\left(v_{*}\right) \leq\left(\Phi_{\lambda}\right)_{v_{*}}^{u_{*}}\left(u_{*}\right) .
$$

The analysis is similar if the opposite inequality holds. By virtute of Claim 2 $u_{*} \in \operatorname{int} C_{+}$is a local minimizer of $\left(\Phi_{\lambda}\right)_{v_{*}}^{u_{*}}$. So, we can find $\rho \in(0,1)$ with $\rho<\left\|v_{*}-u_{*}\right\|$, such that

$$
\left(\Phi_{\lambda}\right)_{v_{*}}^{u_{*}}\left(v_{*}\right) \leq\left(\Phi_{\lambda}\right)_{v_{*}}^{u_{*}}\left(u_{*}\right)<\inf \left\{\left(\Phi_{\lambda}\right)_{v_{*}}^{u_{*}}(u):\left\|u-u_{*}\right\|=\rho\right\}=\eta_{\rho}
$$

Recall that $\left(\Phi_{\lambda}\right)_{v_{*}}^{u_{*}}$ is coercive and so it is easy to prove that it satisfies the Ccondition. This fact and (4.5) permit the use of Theorem 2.1 (the mountain pass theorem). So, we can find $y_{0} \in W^{1, p}(\Omega)$ such that

$$
\begin{gathered}
\left(\Phi_{\lambda}\right)_{v_{*}}^{u_{*}}\left(v_{*}\right) \leq\left(\Phi_{\lambda}\right)_{v_{*}}^{u_{*}}\left(u_{*}\right)<\eta_{\rho} \leq\left(\Phi_{\lambda}\right)_{v_{*}}^{u_{*}}\left(y_{0}\right), \\
\left(\Phi_{\lambda}\right)_{v_{*}}^{\prime u_{*}}\left(y_{0}\right)=0 .
\end{gathered}
$$

From (4.6), we see that $y_{0} \notin\left\{v_{*}, u_{*}\right\}$. From (4.7), see also Lemma 2.6, we have that $y_{0} \in K_{\left(\Phi_{\lambda} u_{* *}^{u_{*}}\right.}$ is also a critical point of $\Phi_{\lambda}$. Hence $y_{0} \in C^{1}(\bar{\Omega})$ is also a solution of problem $\left(\mathrm{P}_{\lambda}\right)$.

Since $y_{0}$ is also a critical point of $\left(\Phi_{\lambda}\right)_{v_{*}}^{u_{*}}$ of mountain pass type, we have

$$
C_{1}\left(\left(\Phi_{\lambda}\right)_{v_{*}}^{u_{*}}, y_{0}\right) \neq 0
$$

On the other hand from Proposition 4.2, we have

$$
C_{1}\left(\left(\Phi_{\lambda}\right)_{v_{*}}^{u_{*}}, 0\right)=0, \quad \text { for all } k \geq 0 .
$$

Comparing (4.8) and (4.9), we infer that $y_{0} \neq 0$. Since $y_{0} \in\left[v_{*}, u_{*}\right]$ and $y_{0} \notin$ $\left\{u_{*}, v_{*}, 0\right\}$, by virtue of the extremality of $u_{*}$ and $v_{*}$, we conclude that $y_{0} \in C^{1}(\bar{\Omega})$ is a nodal solution of $\left(\mathrm{P}_{\lambda}\right)$ with $\lambda \in\left(0, \lambda^{*}\right)$.

\section{Semilinear problems}

In this section, we deal with the semilinear equation (i.e. $p=2$ ). With respect to the case $p \neq 2$, exploiting some ideas introduced in [21], under stronger regularity condition on the perturbation $f(z, \cdot)$ and using Morse theory, we generate a sixth nontrivial solution but we are unable to specify its sign. 
The problem under consideration, is the following:

$$
\begin{cases}-\Delta u+\beta u=\lambda|u|^{q-2} u+f(z, u) & \text { in } \Omega, \\ \frac{\partial u}{\partial n}=0 & \text { on } \partial \Omega,\end{cases}
$$

with $1<q<2$ and $\lambda>0$. The hypotheses on the perturbation $f(z, x)$ are the following:

$\left(\mathrm{H}_{2}\right) f: \Omega \times \mathbb{R} \rightarrow \mathbb{R}$ is a measurable function such that for almost all $z \in \Omega$, $f(z, 0)=0, f(z, \cdot) \in C^{1}(\mathbb{R})$ and

(a) there exists $2 \leq r<2^{*}$ such that $\left|f_{x}^{\prime}(z, x)\right| \leq \alpha(z)\left(1+|x|^{r-2}\right)$ for almost all $z \in \Omega$, all $x \in \mathbb{R}$ with $\alpha \in L^{\infty}(\Omega)_{+}$,

(b) there exist integer $m \geq 2$ and functions $\vartheta, \widehat{\vartheta} \in L^{\infty}(\Omega)$ such that

$$
\widehat{\lambda}_{m}(\beta) \leq \vartheta(z) \leq \widehat{\vartheta}(z) \leq \widehat{\lambda}_{m+1}(\beta) \quad \text { a.e. in } \Omega, \quad \widehat{\lambda}_{m}(\beta) \neq \vartheta, \quad \widehat{\lambda}_{m+1}(\beta) \neq \widehat{\vartheta}
$$

and

$$
\vartheta(z) \leq \liminf _{x \rightarrow \pm \infty} \frac{f(z, x)}{x} \leq \limsup _{x \rightarrow \pm \infty} \frac{f(z, x)}{x} \leq \widehat{\vartheta}(z)
$$

uniformly for almost all $z \in \Omega$,

(c) there exist $\xi_{0}>0$ and $\tau \in(q, 2]$ such that

$$
\xi_{0} \leq \liminf _{x \rightarrow \pm \infty} \frac{f(z, x) x-2 F(z, x)}{|x|^{\tau}} \quad \text { uniformly for a.a. } z \in \Omega,
$$

(d) there exist $\delta_{0} \in(0,1)$ and $r>2$ such that

$0 \leq f(z, x) \leq c_{1}|x|^{r} \quad$ for a.a. $z \in \Omega$, all $|x| \leq \delta_{0}$ and some $c_{1}>0$

and for every $\mu \in\left(0, \delta_{0}\right)$, we can find $\xi_{\mu}>0$ such that

$$
\xi_{\mu} \leq|f(z, x)| \text { for a.a. } z \in \Omega \text {, all } x \in\left[\mu, \delta_{0}\right] .
$$

REMARK 5.1. Evidently, hypothesis $\left(\mathrm{H}_{2}\right)(\mathrm{d})$ implies that $f_{x}^{\prime}(z, 0)=0$ for almost all $z \in \Omega$. Also, from hypothesis $\left(\mathrm{H}_{2}\right)(\mathrm{a})$ and the mean value theorem, we see that in this case hypothesis $\left(\mathrm{H}_{1}\right)(\mathrm{e})$ is automatically satisfied.

For $\lambda>0$, let $\Phi_{\lambda}: H^{1}(\Omega) \rightarrow \mathbb{R}$ be the energy functional for problem $\left(\mathrm{S}_{\lambda}\right)$ defined by

$$
\Phi_{\lambda}(u)=\frac{1}{2} \sigma(u)-\frac{\lambda}{q}\|u\|_{q}^{q}-\int_{\Omega} F(z, u(z)) d z, \quad \text { for all } u \in H^{1}(\Omega) .
$$

In this case

$$
\sigma(u)=\|D u\|_{2}^{2}+\int_{\Omega} \beta(z) u(z)^{2} d z, \quad \text { for all } u \in H^{1}(\Omega) .
$$

Clearly, $\Phi_{\lambda} \in C^{2}\left(H^{1}(\Omega) \backslash\{0\}\right)$. For the problem $\left(\mathrm{S}_{\lambda}\right)$, we have the following multiplicity theorem: 
TheOREm 5.2. If hypotheses $\mathrm{H}(\beta)$ and $\left(\mathrm{H}_{2}\right)$ hold, then there exists $\lambda^{*}>0$ such that for all $\lambda \in\left(0, \lambda^{*}\right)$ problem $\left(\mathrm{S}_{\lambda}\right)$ has at least six nontrivial solutions:

$$
\begin{gathered}
u_{0}, \widehat{u} \in \operatorname{int} C_{+}, \quad u_{0} \leq \widehat{u}, \quad u_{0} \neq \widehat{u}, \\
v_{0}, \widehat{v} \in-\operatorname{int} C_{+}, \quad \widehat{v} \leq v_{0}, \quad v_{0} \neq \widehat{v}, \\
y_{0} \in \operatorname{int}_{C^{1}(\bar{\Omega})}\left[v_{0}, u_{0}\right] \quad \text { nodal } \quad \text { and } \widehat{y} \in C^{1}(\bar{\Omega}) .
\end{gathered}
$$

Proof. From Theorem 4.3, we already have five nontrivial solutions:

$$
\begin{gathered}
u_{0}, \widehat{u} \in \operatorname{int} C_{+}, \quad u_{0} \leq \widehat{u}, \quad u_{0} \neq \widehat{u}, \\
v_{0}, \widehat{v} \in-\operatorname{int} C_{+}, \quad \widehat{v} \leq v_{0}, \quad v_{0} \neq \widehat{v}, \\
\text { and } y_{0} \in \operatorname{int}_{C^{1}(\bar{\Omega})}\left[v_{0}, u_{0}\right] \text { nodal. }
\end{gathered}
$$

From Proposition 3.1, we know that solutions $u_{0}$ and $v_{0}$ are local minimizers of the energy functional $\Phi_{\lambda}$. So, we have

$$
C_{k}\left(\Phi_{\lambda}, u_{0}\right)=C_{k}\left(\Phi_{\lambda}, v_{0}\right)=\delta_{k, 0} \mathbb{Z}, \quad \text { for all } k \geq 0 .
$$

Let $\varrho=\|\widehat{u}\|_{\infty}$ and let $\xi_{\varrho}>0$ be such that for almost all $z \in \Omega, x \rightarrow$ $f(z, x)+\xi_{\varrho} x^{p-1}$ is nondecreasing on $[0, \varrho]$. We have

$$
\begin{array}{rlrl}
-\Delta u_{0}(z) & +\left(\beta(z)+\xi_{\varrho}\right) u_{0}(z) & & \\
= & \lambda u_{0}^{q-1}(z)+f\left(z, u_{0}(z)\right)+\xi_{\varrho} u_{0}(z) & \\
\leq & \lambda \widehat{u}^{q-1}(z)+f(z, \widehat{u}(z))+\xi_{\varrho} \widehat{u}(z) & & \left(\text { since } u_{0} \leq \widehat{u}\right) \\
= & -\Delta \widehat{u}(z)+\left(\beta(z)+\xi_{\varrho}\right) \widehat{u}(z) & & \text { a.e. in } \Omega .
\end{array}
$$

From this, one has

$$
\Delta\left(\widehat{u}-u_{0}\right)(z) \leq\left(\|\beta\|_{\infty}+\xi_{\varrho}\right)\left(\widehat{u}-u_{0}\right)(z) \text { a.e. in } \Omega,
$$

and owing to [26], $\widehat{u}-u_{0} \in \operatorname{int} C_{+}$. We introduce the set

$$
\left[u_{0}\right)=\left\{u \in W^{1, p}(\Omega): u_{0}(z) \leq u(z) \text { a.e. in } \Omega\right\},
$$

and recall that $\left(\Phi_{\lambda}\right)_{0}: W^{1, p}(\Omega) \rightarrow \mathbb{R}$ is the $C^{1}$-functional defined by

$$
\left(\Phi_{\lambda}\right)_{0}(u)=\frac{\sigma(u)}{p}-\int_{\Omega}\left(L_{\lambda}\right)_{0}(z, u(z)) d z, \quad \text { for all } u \in W^{1, p}(\Omega) .
$$

We have

$$
\left.\left(\Phi_{\lambda}\right)_{0}\right|_{\left[u_{0}\right)}=\left.\left(\Phi_{\lambda}\right)_{u_{0}}\right|_{\left[u_{0}\right)}+\xi_{\lambda}^{+}, \quad \text { for some } \xi_{\lambda}^{+} \in \mathbb{R},
$$

$$
\Rightarrow C_{k}\left(\left.\left(\Phi_{\lambda}\right)_{0}\right|_{C^{1}(\bar{\Omega})}, \widehat{u}\right)=C_{k}\left(\left.\left(\Phi_{\lambda}\right)_{u_{0}}\right|_{C^{1}(\bar{\Omega})}, \widehat{u}\right), \quad \text { for all } k \geq 0 .
$$


Moreover, from Palais [23] (or alternatively using the homotopy invariance of the critical groups) we have

$$
\begin{aligned}
C_{k}\left(\left.\left(\Phi_{\lambda}\right)_{0}\right|_{C^{1}(\bar{\Omega})}, \widehat{u}\right) & =C_{k}\left(\left(\Phi_{\lambda}\right)_{0}, \widehat{u}\right), & & \text { for all } k \geq 0, \\
C_{k}\left(\left.\left(\Phi_{\lambda}\right)_{u_{0}}\right|_{C^{1}(\bar{\Omega})}, \widehat{u}\right) & =C_{k}\left(\left(\Phi_{\lambda}\right)_{u_{0}}, \widehat{u}\right), & & \text { for all } k \geq 0 .
\end{aligned}
$$

Similarly, since $\widehat{u} \in \operatorname{int} C_{+}$and $\left.\Phi_{\lambda}\right|_{C_{+}}=\left.\left(\Phi_{\lambda}\right)_{0}\right|_{C_{+}}$, we have

$$
C_{k}\left(\Phi_{\lambda}, \widehat{u}\right)=C_{k}\left(\left(\Phi_{\lambda}\right)_{0}, \widehat{u}\right), \quad \text { for all } k \geq 0 .
$$

Finally, recall that $\widehat{u}$ is a critical point of $\left(\Phi_{\lambda}\right)_{u_{0}}$ of mountain pass type. Therefore

$$
C_{1}\left(\left(\Phi_{\lambda}\right)_{u_{0}}, \widehat{u}\right) \neq 0 .
$$

From (5.2)-(5.6) it follows that $C_{1}\left(\Phi_{\lambda}, \widehat{u}\right) \neq 0$. This, by virtue of Proposition 25 of Bartsch [2], implies that

$$
C_{k}\left(\Phi_{\lambda}, \widehat{u}\right)=\delta_{k, 1} \mathbb{Z}, \quad \text { for all } k \geq 0 .
$$

In a similar way, we show that

$$
C_{k}\left(\Phi_{\lambda}, \widehat{v}\right)=\delta_{k, 1} \mathbb{Z}, \quad \text { for all } k \geq 0 .
$$

Via the maximum principle of Vazquez [26], as before we can show that

$$
\begin{aligned}
u_{0}-y_{0} \in \operatorname{int} C_{+} \quad \text { and } \quad y_{0}-v_{0} & \in \operatorname{int} C_{+}, \\
& \Rightarrow y_{0} \in \operatorname{int}_{C^{1}(\bar{\Omega})}\left[v_{0}, u_{0}\right] .
\end{aligned}
$$

Assuming without any loss of generality that $u_{0}$ and $v_{0}$ are extremal (i.e. $u_{0}=u_{*}$, $v_{0}=v_{*}$, see Proposition 3.5) and using the notation of Section 4, we have

$$
\left.\Phi_{\lambda}\right|_{\left[v_{0}, u_{0}\right]}=\left.\left(\Phi_{\lambda}\right)_{v_{*}}^{u_{*}}\right|_{\left[v_{0}, u_{0}\right]} .
$$

Since $y_{0}$ is a critical point of mountain pass type (see the proof of Theorem 4.3), as above we obtain

$$
C_{k}\left(\Phi_{\lambda}, y_{0}\right)=\delta_{k, 1} \mathbb{Z}, \quad \text { for all } k \geq 0 .
$$

From Proposition 4.1, we have

$$
C_{k}\left(\Phi_{\lambda}, 0\right)=0, \quad \text { for all } k \geq 0 .
$$

Let

$$
\bar{H}_{m}=\bigoplus_{i=1}^{m} E\left(\widehat{\lambda}_{i}(\beta)\right) \quad \text { and } \quad \widehat{H}_{m}=\bar{H}_{m}^{1}=\bigoplus_{i \geq m+1} E\left(\widehat{\lambda}_{i}(\beta)\right) .
$$

We have orthogonal direct sum decomposition $H^{1}(\Omega)=\bar{H}_{m} \oplus \widehat{H}_{m+1}$.

By virtue of hypotheses $\left(\mathrm{H}_{2}\right)(\mathrm{a}),(\mathrm{b})$, given $\varepsilon>0$, we can find $c_{12}=c_{12}(\varepsilon)>0$ such that

$$
F(z, x) \geq \frac{1}{2}(\vartheta(z)-\varepsilon) x^{2}-c_{12}, \quad \text { for a.a. } z \in \Omega, \text { all } x \in \mathbb{R} .
$$


Then, for $u \in \bar{H}_{m}$, we have

$$
\begin{aligned}
\Phi_{\lambda}(u) & =\frac{1}{2} \sigma(u)-\frac{\lambda}{q}\|u\|_{q}^{q}-\int_{\Omega} F(z, u) d z \\
& \leq \frac{1}{2}\left[\sigma(u)-\int_{\Omega} \vartheta u^{2} d z+\varepsilon\|u\|^{2}\right]-\frac{\lambda}{q}\|u\|_{q}^{q}+c_{12}|\Omega|_{N} \\
& \leq \frac{1}{2}\left[\varepsilon-c_{13}\right]\|u\|^{2}+c_{12}|\Omega|_{N}, \quad \text { for some } c_{13}>0 .
\end{aligned}
$$

Choosing $\varepsilon \in\left(0, c_{13}\right)$, we infer that

$$
\Phi_{\lambda}(u) \rightarrow-\infty \quad \text { as }\|u\| \rightarrow \infty, u \in \bar{H}_{m} .
$$

On the other hand, again from hypotheses $\left(\mathrm{H}_{2}\right)(\mathrm{a}),(\mathrm{b})$, given $\varepsilon>0$, we can find $c_{14}=c_{14}(\varepsilon)$ such that

$$
F(z, x) \leq \frac{1}{2}(\widehat{\vartheta}(z)+\varepsilon) x^{2}+c_{14}, \quad \text { for a.a. } z \in \Omega, \text { all } x \in \mathbb{R} .
$$

Then, for $u \in \bar{H}_{m+1}$, we have

$$
\begin{aligned}
\Phi_{\lambda}(u) & =\frac{1}{2} \sigma(u)-\frac{\lambda}{q}\|u\|_{q}^{q}-\int_{\Omega} F(z, u) d z \\
& \geq \frac{1}{2}\left[c_{15}-\varepsilon\right]\|u\|^{2}-c_{16}\|u\|^{q}-c_{14}|\Omega|_{N}, \quad \text { for some } c_{15}, c_{16}>0 .
\end{aligned}
$$

Choosing $\varepsilon \in\left(0, c_{15}\right)$, and since $q<2$, we infer that

$$
\Phi_{\lambda}(u) \rightarrow+\infty \quad \text { as }\|u\| \rightarrow \infty, u \in \bar{H}_{m+1} .
$$

Recall that $\Phi_{\lambda}$ satisfies the C-condition. This fact and (5.14), (5.15) permit the use of Proposition 3.8 of Bartsch and Li [3]. So, we have

$$
C_{d_{m}}\left(\Phi_{\lambda}, \infty\right) \neq 0, \quad \text { where } d_{m}=\operatorname{dim} \bar{H}_{m} \geq 2 \quad(\text { recall } m \geq 2) .
$$

This means that we can find $\widehat{y} \in K_{\Phi_{\lambda}}$ such that

$$
C_{d_{m}}\left(\Phi_{\lambda}, \widehat{y}\right) \neq 0 .
$$

Comparing (5.16) with (5.1), (5.7), (5.8), (5.10), (5.11), we infer that

$$
\widehat{y} \in K_{\Phi_{\lambda}} \backslash\left\{0, u_{0}, v_{0}, \widehat{u}, \widehat{v}, y_{0}\right\},
$$

then $\widehat{y} \in C^{1}(\bar{\Omega})$ is the sixth nontrivial solution of $\left(\mathrm{S}_{\lambda}\right), \lambda \in\left(0, \lambda^{*}\right)$.

REMARK 5.3. It is an interesting open problem to see whether $\widehat{y}$ is nodal.

Acknowledgements. The authors have been supported by the Gruppo Nazionale per l'Analisi Matematica, la Probabilità e le loro Applicazioni (GNAMPA) of the Istituto Nazionale di Alta Matematica (INdAM). The authors wish to thank the referee for his/her remarks. 


\section{REFERENCES}

[1] S. Aizicovici, N. Papageorgiou And V. Staicu, Existence of multiple solutions with precise sign information for superlinear Neumann problems, Ann. Mat. Pura Appl. (4) 188 (2009), no. 4, 679-719.

[2] T. BARTSCH, Critical point theory on partially ordered Hilbert spaces, J. Funct. Anal. 186 (2001), no. 1, 117-152.

[3] T. Bartsch AND S. LI, Critical point theory for asymptotically quadratic functionals and applications to problems with resonance, Nonlinear Anal. 28 (1997), no. 3, 419-441.

[4] F.O. DE PAIVA AND E. MAssa, Multiple solutions for some elliptic equations with a nonlinearity concave at the origin, Nonlinear Anal. 66 (2007), no. 12, 2940-2946.

[5] J.I. DAZ AND J.E. SAÁ, Existence et unicité de solutions positives pour certaines equations elliptiques quasilinéaires, C.R. Acad. Sci. Paris Sér. I Math. 305 (1987), no. 12, 521-524.

[6] N. Dunford and J. Schwartz, Linear Operators, Wiles-Interscience, New York (1958).

[7] L. Gasinski And N.S. Papageorgiou, Nonlinear Analysis, Ser. Math. Anal. Appl. 9, Chapman and Hall/CRC Press, Boca Raton, 2006.

[8] _ Nonlinear elliptic equations with singular terms and combined nonlinearities, Ann. H. Poincaré 13 (2012), no. 3, 481-512.

[9] _ A pair of positive solutions for the Dirichlet $p(z)$-Laplacian with concave and convex nonlinearities, J. Global Optim. 56 (2013), no. 4, 1347-1360.

[10] Z. Guo And Z. Zhang, $W^{1, p}$ versus $C^{1}$ local minimizers and multiplicity results for quasilinear elliptic equations, J. Math. Anal. Appl. 286 (2003), no. 1, 32-50.

[11] S. Hu AND N. PAPAGEORGIOU, Nonlinear Neumann equations driven by a nonhomogeneous differential operator, Commun. Pure Appl. Anal. 10 (2011), no. 4, 1055-1078.

[12] _ Double resonance for Dirichlet problems with unbounded indefinite potential and combined nonlinearities, Commun. Pure Appl. Anal. 11 (2012), no. 5, 2005-2021.

[13] Q. JIU AND J. SU, Existence and multiplicity results for Dirichlet problems with pLaplacian, J. Math. Anal. Appl. 281 (2003), no. 2, 587-601.

[14] S. Kyritsi and N.S. Papageorgiou, Pairs of positive solutions for p-Laplacian equations with combined nonlinearities, Commun. Pure Appl. Anal. 8 (2009), no. 3, 1031-1051.

[15] G. Lieberman, Boundary regularity for solutions of degenerate elliptic equations, Nonlinear Anal. 12 (1988), 1203-1219.

[16] S. LI, S. Wu AND H.Z. Zhou, Solutions to semilinear elliptic problems with combined nonlinearities, J. Differential Equations 185 (2002), no. 1, 200-224.

[17] S.A. Marano and N.S. Papageorgiou, Positive solutions to a Dirichlet problem with $p$-Laplacian and concave-convex nonlinearity depending on a parameter, Commun. Pure Appl. Anal. 12 (2013), no. 2, 815-829.

[18] D. Motreanu and N.S. Papageorgiou, Multiple solutions for nonlinear Neumann problems driven by a nonhomogeneous differential operator, Proc. Amer. Math. Soc. 139 (2011), no. 10, 3527-3535.

[19] D. Motreanu, V.V. Motreanu and N.S. Papageorgiou, On p-Laplace equations with concave terms and asymmetric perturbations, Proc. Roy. Soc. Edinburgh Sect. A 141 (2011), no. 1, 171-192.

[20] _ Multiple constant sign and nodal solutions for nonlinear Neumann eigenvalue problems, Ann. Sc. Norm. Super. Pisa Cl. Sci. (5) 10 (2011), no. 3, 729-755.

[21] _ On resonant Neumann problems, Math. Ann. 354 (2012), no. 3, 1117-1145.

[22] D. Mugnai and N.S. Papageorgiou, Resonant nonlinear Neumann problems with indefinite weight, Ann. Sc. Norm. Sup. Pisa Cl. Scu. Vol. XI, 4 (2012), 729-788. 
[23] R. PAlais, Homotopy theory of indefinite dimensional manifolds, Topology 5 (1966), $1-16$.

[24] N.S. Papageorgiou And G. Smyrlis, Positive solutions for nonlinear Neumann problems with concave and convex terms, Positivity 16 (2012), no. 2, 271-296.

[25] K. PERERA, Multiplicity results for some elliptic problems with concave nonlinearities, J. Differential Equations 140 (1997), 133-141.

[26] J.L. VÁzQUEZ, A strong maximum principle for some quasilinear elliptic equations, Appl. Math. Optim. 12 (1984), 191-202.

[27] S.P. WU AND H. YANG, A class of resonant elliptic problems with sublinear nonlinearity at origin and at infinity, Nonlinear Anal. 45 (2001), 925-935.

Pasquale Candito

Department of Engineering

University of Reggio Calabria

Via Graziella (Feo Di Vito)

Reggio Calabria, ITALY

E-mail address: pasquale.candito@unirc.it

Giuseppina D'Aguí

Department DICIEAMA

University of Messina, Contrada Di Dio (S. Agata)

Messina, ITALY

E-mail address: dagui@unime.it

Nikolaos S. Papageorgiou

National Technical University

Department of Mathematics

Zografou Campus

Athens, 45780 GREECE

E-mail address: npapg@math.ntua.gr 